\title{
Valoración del crecimiento J la alimentación durante la lactancia s la primera infancia en atención primaria
}

José Luis Olivares Lopez

Gerardo Rodríguez Martínez

Pilar Samper Villagrasa
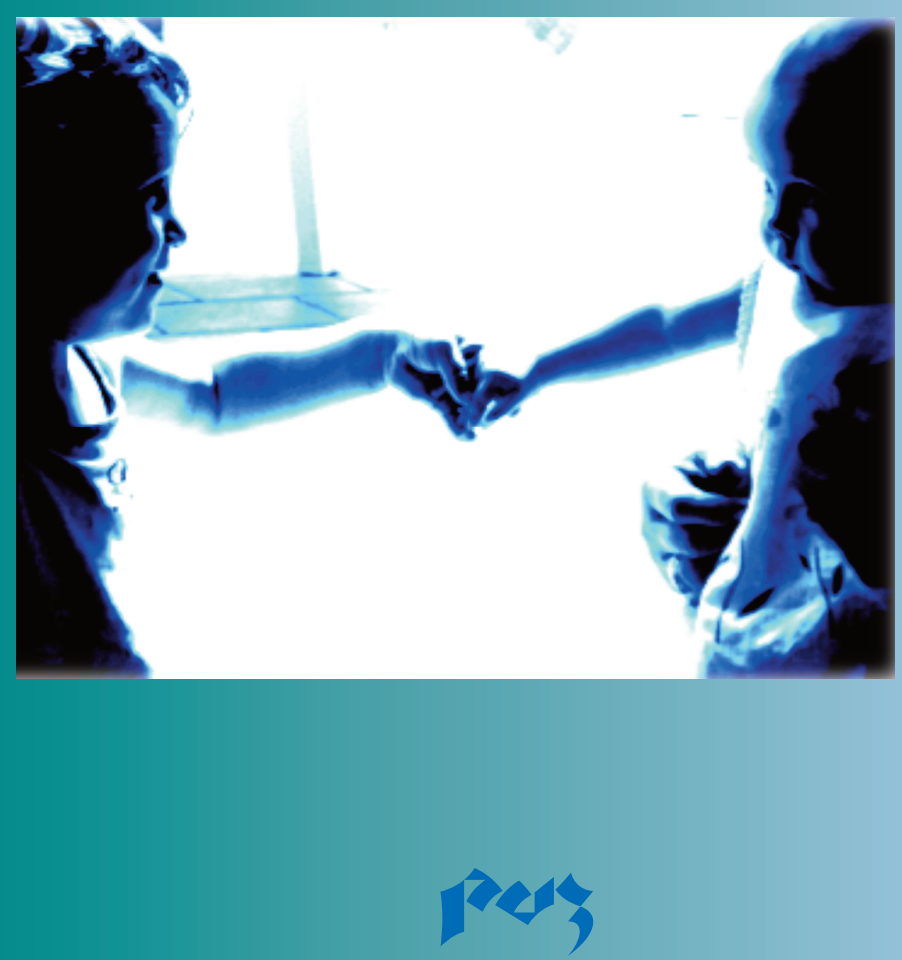

Prensas Universitarias de Zaragoza 



\section{VALORACIÓN DEL CRECIMIENTO Y LA ALIMENTACIÓN}

DURANTE LA LACTANCIA Y LA PRIMERA INFANCIA EN ATENCIÓN PRIMARIA 



\section{VALORACIÓN DEL CRECIMIENTO Y LA ALIMENTACIÓN \\ DURANTE LA LACTANCIA \\ Y LA PRIMERA INFANCIA \\ EN ATENCIÓN PRIMARIA}

José Luis Olivares López

Gerardo Rodríguez Martínez

Pilar Samper Villagrasa

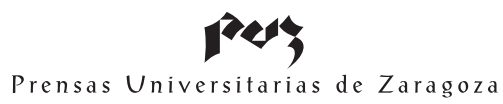


(C) José Luis Olivares López, Gerardo Rodríguez Martínez y Pilar Samper Villagrasa

(C) De la presente edición, Prensas Universitarias de Zaragoza

1. a edición, 2009

Prensas Universitarias de Zaragoza

Edificio de Ciencias Geológicas

c/ Pedro Cerbuna, 12, 50009 • Zaragoza, España

Tel.: 976761 330. Fax: 976761063

puz@unizar. http://puz.unizar.es

Impreso en España

Imprime: Servicio de Publicaciones. Universidad de Zaragoza

ISBN: 978-84-92774-36-4

D.L.: Z-3636/2009 


\title{
Valoración del crecimiento y la alimentación durante la lactancia y la primera infancia en atención primaria
}

\author{
PROYECTO CALINA \\ (Crecimiento y Alimentación durante la Lactancia y la primera Infancia \\ en Niños Aragoneses)
}

\author{
José Luis Olivares López. \\ Catedrático de Pediatría. Universidad de Zaragoza \\ Jefe del Servicio de Pediatría del Hospital Clínico Universitario Lozano Blesa
}

\author{
Gerardo Rodríguez Martínez \\ Profesor Titular de Pediatría. Universidad de Zaragoza \\ Servicio de Pediatría. Hospital Clínico Universitario Lozano Blesa \\ Instituto Aragonés de Ciencias de la Salud
}

Pilar Samper Villagrasa

Profesora Colaboradora. Área de Pediatría. Universidad de Zaragoza 



\section{AUTORES COLABORADORES}

(Por orden alfabético)

Alijarde Lorente, Roberto

Pediatra

C.S. Perpetuo Socorro. Huesca

Álvarez Sauras, M. ${ }^{a}$ Luisa

Nutricionista

Universidad de Zaragoza

Atance Melendo, Esther

Enfermera

C.S. Valdefierro. Zaragoza

Balagué Clemos, Concepción

Enfermera

C.S. Torrero-La Paz. Zaragoza

Bartolomé Lalanza, M. ${ }^{\mathrm{a}}$ Lucía

Pediatra

C.S. Actur Oeste (Amparo Poch). Zaragoza

Blasco Pérez-Aramendia, M. a Jesús

Pediatra

C.S. Valdefierro. Zaragoza
Adivinación Herrero, Dori

Enfermera

C.S. Teruel Centro. Teruel

Álvarez Otazu, M. ${ }^{a}$ Jesús

Enfermera

C.S. Borja. Zaragoza

Arana Navarro, Teresa

Pediatra

C.S. Sagasta-Miraflores. Zaragoza

Ayerza Casas, Ariadna

Pediatra

Hospital Clínico Universitario

Lozano Blesa. Zaragoza

Baños Ledesma, M. ${ }^{a}$ Victoria

Enfermera

C.S. Tarazona. Zaragoza

Bartrés Soler, Teresa

Pediatra

C.S. Las Fuentes Norte. Zaragoza

Broto Cosculluela, Purificación

Enfermera

C.S. Perpetuo Socorro. Huesca 
Cabañas Bravo, M. ${ }^{a}$ Jesús

Pediatra

C.S. Delicias Sur. Zaragoza

Cebrián Gimeno, M. ${ }^{a}$ Inmaculada

Pediatra

C.S. Torrero-La Paz. Zaragoza

Chicote Abadía, M. ${ }^{a}$ Begoña

Enfermera

C.S. Actur Oeste (Amparo Poch). Zaragoza

Elviro Mayoral, Luis Carlos

Pediatra

C.S. Actur Oeste (Amparo Poch). Zaragoza

Falcón Polo, Ángeles

Enfermera.

C.S. Actur Norte. Zaragoza

Fondevilla Pérez, M. ${ }^{\text {a }}$ Teresa

Enfermera

C.S. Sagasta-Ruiseñores. Zaragoza

Fuertes Domínguez, Amparo

Pediatra

C.S. Delicias Sur. Zaragoza

Galán Rico, José

Pediatra

C.S. Teruel Centro. Teruel

\section{Cáncer Raginal, Rosa}

Enfermera

C.S. Torrero-La Paz. Zaragoza

\section{Cenarro Guerrero, Teresa}

Pediatra

C.S. Sagasta-Ruiseñores. Zaragoza

\section{Crespo Mainar, María Cleofé}

Enfermera

C.S. Delicias Sur. Zaragoza

\section{Esteban Herréiz, Concha}

Enfermera

C.S. Delicias Sur. Zaragoza

Feliz de Vargas Pastor, Jesús

Pediatra

C.S. Teruel Ensanche. Teruel

Forés Catalá, M. ${ }^{a}$ Desamparados

Enfermera

C.S. Las Fuentes Norte. Zaragoza

Fuertes Fernández-Espinar, Jorge

Pediatra

C.S. Perpetuo Socorro. Huesca

Galbe Sánchez-Ventura, José

Pediatra

C.S. Torrero-La Paz. Zaragoza 


\section{Gallego Pérez, Matilde}

Enfermera

C.S. Valdefierro. Zaragoza

\section{García Vera, César}

Pediatra

C.S. Sagasta-Ruiseñores. Zaragoza

\section{Gila Gajón, M. ${ }^{a}$ Asunción}

Enfermera

C.S. Sagasta-Ruiseñores. Zaragoza

\section{Laín Ara, M. ${ }^{a}$ Lourdes}

Enfermera

C.S. Jaca. Huesca

\section{Lalana Josa, M. ${ }^{a}$ Pilar}

Pediatra

C.S. Borja. Zaragoza

Lasarte Velillas, Juan José

Pediatra

C.S. Zuera. Zaragoza

\section{Magallón Botalla, Rosa}

Médico de familia

Instituto Aragonés de Ciencias de la Salud

Coordinadora del Grupo Aragonés de Investigación en Atención Primaria (GAIAP)

\section{García Sánchez, Nuria}

Pediatra

C.S. Delicias Sur. Zaragoza

\section{Garín Moreno, Ana-Luz}

Pediatra

C.S. Las Fuentes Norte. Zaragoza

Júdez Molina, Carmen

Enfermera

C.S. Actur Norte. Zaragoza

Lalaguna Puértolas, M. ' Jesús

Enfermera

C.S. Jaca. Huesca

\section{Lambán Casamayor, Elisa}

Enfermera

C.S. Las Fuentes Norte. Zaragoza

Lostal Gracia, M. ${ }^{\text {a Isabel }}$

Pediatra

C.S. Actur Oeste (Amparo Poch) Zaragoza

\section{Marco Olloqui, Mónica}

Pediatra

C.S. Valdefierro. Zaragoza 
Marín Ibáñez, M. ${ }^{a}$ Pilar

Enfermera

C.S. Las Fuentes Norte. Zaragoza

Martínez Espligares, Laura

Pediatra

C.S. Tarazona. Zaragoza

\section{Moneo Hernández, Isabel}

Pediatra

C.S. Las Fuentes Norte. Zaragoza

Moreno Aznar, Luis A.

Prof. Titular Universidad

Universidad de Zaragoza

Muñoz Jalle, Elena

Pediatra

Hospital Clínico Universitario Lozano Blesa

Olivares López, José Luis

Catedrático de Pediatría. Universidad de Zaragoza

Hospital Clínico Universitario Lozano Blesa

\section{Pinilla Fuentes, José Antonio}

Pediatra

C.S. Jaca. Huesca

\section{Martínez Bueno, José Luis}

Enfermero

C.S. Zuera. Zaragoza

\section{Mengual Gil, José M. ${ }^{\text {a }}$}

Pediatra

Instituto Aragonés de Ciencias de la Salud

Director del Área de Transferencia del

Conocimiento

\section{Montaner Cosa, Mercedes}

Enfermera

C.S. Teruel Ensanche. Teruel

\section{Muñoz Campos, Ana Isabel}

Pediatra

C.S. Teruel Ensanche. Teruel

\section{Navarro Serrano, Eva María}

Pediatra

C.S. Teruel Centro. Teruel

\section{Pardos Martínez, Luis Carlos}

Pediatra

C.S. Perpetuo Socorro. Huesca

\author{
Puig García, Carmen \\ Pediatra \\ C.S. Actur Oeste (Amparo Poch) Zaragoza
}


Puyuelo del Val, Pascual

Pediatra

C.S. Jaca. Huesca

\section{Rodríguez Martínez, Gerardo}

Profesor Titular de Pediatría. Universidad de Zaragoza Hospital Clínico Universitario Lozano Blesa

Instituto Aragonés de Ciencias de la Salud

\section{Sánchez Gimeno, Javier}

Pediatra

C.S. Sagasta-Ruiseñores. Zaragoza

\section{Sebastián Bonel, M. ${ }^{\mathrm{a}}$ Flor}

Enfermera

C.S. Perpetuo Socorro. Huesca

\section{Soria Martínez, Leticia}

Enfermera

C.S. Tarazona. Zaragoza

Traver Cabrera, Pilar

Pediatra

C.S. Zuera. Zaragoza

\section{Viñas Viamonte, $M{ }^{a}{ }^{a}$ Carmen}

Enfermera

C.S. Actur Norte. Zaragoza

\section{Redondo Cuerpo, M. ${ }^{\text {a }}$ Victoria}

Enfermera

C.S. Actur Oeste (Amparo Poch) Zaragoza

\section{Samper Villagrasa, Pilar}

Profesora Colaboradora. Área de Pediatría

Universidad de Zaragoza

\section{Sánchez Zapater, Asunción}

Pediatra

C.S. Actur Oeste (Amparo Poch) Zaragoza

Solans Bascuas, M. ${ }^{\mathrm{a}}$ Teresa

Pediatra

C.S. Delicias Sur. Zaragoza

\section{Traín del Val, Ana}

Enfermera

C.S. Sagasta-Miraflores. Zaragoza

Verón Jiménez, M. ${ }^{a}$ Asunción

Enfermera

C.S. Delicias Sur. Zaragoza

\section{Zarazaga Germes, Gregorio}

Pediatra

C.S. Torrero-La Paz. Zaragoza 

El equipo investigador agradece el apoyo prestado por el Instituto Aragonés de Ciencias de la Salud y por el Instituto de Salud Carlos III (Ministerio de Ciencia e Innovación; PI080559) en la planificación y financiación del Proyecto CALINA (Crecimiento y alimentación durante la lactancia y la primera infancia en niños aragoneses).

Dedicamos el presente estudio a todos los niños y padres que han participado de manera ejemplar. A todos ellos, ;muchas gracias! 



\section{PREFACIO}

Un buen estado nutricional y un crecimiento adecuado durante las primeras etapas de la vida mejoran las expectativas de salud a corto y largo plazo. El presente proyecto se planificó precisamente para estudiar el patrón de crecimiento actual y las pautas de alimentación de una muestra representativa de niños aragoneses hasta los 24 meses de edad. Así mismo, con los datos longitudinales obtenidos, se podrán determinar los factores prenatales, postnatales, culturales y psico-sociales que puedan influir en el estado nutricional de nuestra población. El proyecto CALINA (crecimiento y alimentación durante la lactancia y la primera infancia en niños aragoneses) pretende describir la normal variabilidad del crecimiento y alimentación en los niños menores de 24 meses de nuestro entorno; obteniendo datos actualizados que sirvan para la detección de grupos de riesgo nutricional y como punto de partida de futuros proyectos de intervención poblacional.

El proyecto CALINA está diseñado para su desarrollo multicéntrico en Atención Primaria, con la participación de un equipo multidiscipinar compuesto por profesionales del Área de Pediatría de la Universidad de Zaragoza, del Instituto Aragonés de Ciencias de la Salud y de Pediatría de Atención Primaria. Como resultado, se ha conseguido un proyecto científico bien planificado que cuenta con el respaldo institucional, es factible desde los Centros de Salud, con unos objetivos de gran enfoque práctico y que seguro obtendrá resultados útiles desde el punto de vista asistencial e investigador. El estudio CALINA materializa las inquietudes y el interés común de los miembros de un equipo que anhela avanzar en un área del conocimiento tan importante como es la nutrición, el desarrollo y el crecimiento del niño; con datos actualizados que reflejen la realidad de nuestro entorno. 
Ha confluido una serie de circunstancias que han permitido la puesta en marcha de un proyecto como este, de diseño longitudinal, en alrededor de 2000 niñas y niños aragoneses. En primer lugar, la formación de un grupo llamado CORE con los componentes que inicialmente representaron a las diversas instituciones implicadas y que coordinaron el diseño y el reclutamiento del resto del equipo. En segundo lugar, el apoyo económico del Instituto de Salud Carlos III (Ministerio de Ciencia e Innovación) y el metodológico del Instituto Aragonés de Ciencias de la Salud (Gobierno de Aragón), siendo este último también vehículo de la solicitud institucional y de la posterior gestión económica del proyecto. Finalmente, la estructura asistencial de los Centros de Salud de Atención Primaria que permite incorporar el protocolo de estudio del proyecto CALINA en su quehacer diario dentro del «Seguimiento del Niño Sano», con los aproximadamente 60 profesionales (personal facultativo de pediatría y de enfermería) que estarán implicados directamente en su desarrollo y con los que lo harán también indirectamente.

El proyecto CALINA supone un hito evolutivo en los estudios planificados desde Pediatría de Atención Primaria en Aragón (incluso en el resto de España) por sus características, diseño, calidad científica y por la importancia que puedan alcanzar sus resultados. El estudio CALINA supone un desafío para todos los componentes del equipo investigador y, a su vez, una actividad atractiva para un grupo de profesionales ilusionados con la idea de progresar aportando su esfuerzo y de complementar su actividad, en su mayor parte asistencial, con unas pinceladas de ciencia tan importante para el avance del saber. 


\section{MEMORIA DEL PROYECTO CALINA}

\subsection{INTRODUCCIÓN Y JUSTIFICACIÓN}

\section{Crecimiento}

El crecimiento se define como el aumento progresivo en el número de células del organismo y en la adquisición de funciones cada vez más específicas por parte de los diversos órganos, tejidos y sistemas que lo integran. A diferencia del adulto, el niño se encuentra constantemente en crecimiento y desarrollo físico y mental desde las primeras etapas de la vida $(1,2)$. El crecimiento está regulado por múltiples genes, factores de trascripción, hormonas, factores de crecimiento y elementos de la homeostasis celular. La alimentación es la encargada de aportar la energía y los nutrientes necesarios para el crecimiento óptimo durante la infancia y la adolescencia, pudiendo aparecer alteraciones si el aporte nutricional es inadecuado, ya sea por exceso o por defecto (2). Además de aportar materiales plásticos y energéticos que permitan un funcionalismo óptimo, la cantidad y composición de la dieta también tiene efectos reguladores sobre el crecimiento, modulando la expresión de determinados genes, influenciando la acción de los factores de crecimiento y, en definitiva, induciendo resultados fenotípicos a partir de la exposición a una dieta determinada (2). Por ejemplo, las alteraciones nutricionales y la propia composición de la dieta durante la época intrauterina y postnatal precoz son capaces de modular el crecimiento y la distribución del tejido adiposo, el crecimiento del compartimento no graso, la termogénesis y la sensibilidad a la insulina $(3,4)$. Este fenómeno se conoce como programación metabólica. 
Se ha demostrado que la ganancia rápida de peso desde el nacimiento hasta los dos años de vida es un factor de riesgo claro de aparición posterior de obesidad, alteraciones cardiometabólicas y diabetes mellitus tipo 2 (4). Esto ocurre especialmente en los nacidos tras un periodo de escasez nutricional intrauterina y en los que han padecido desnutrición durante la época postnatal precoz, experimentando posteriormente un fenómeno de crecimiento rápido durante las siguientes semanas o meses, también llamado crecimiento recuperador o catch up (3-7). Así pues, el peso al nacimiento y el crecimiento durante los primeros meses de la vida pueden modificar aspectos de la composición corporal (excesivo aumento de la adiposidad y de la grasa intraabdominal, y un escaso aumento del compartimento no graso) y asociarse a la aparición de alteraciones metabólicas (principalmente por el desarrollo de insulino resistencia) $(4,6)$.

Entre otros, los principales factores que han mostrado evidencia científica al relacionarse con la aparición posterior de obesidad, en estudios longitudinales fiables o tras metaanálisis rigurosos son: el peso al nacer, hábito tabáquico en la madre durante la gestación, crecimiento intrauterino restringido con catch-up postnatal, ingesta elevada de proteína y energía en los primeros meses de vida, alta tasa de crecimiento durante los primeros 12 meses de vida, ausencia o poco tiempo de lactancia materna, rebote de adiposidad temprano e ingesta de bebidas azucaradas (4-7).

La normalidad o la alteración del patrón de crecimiento de un sujeto se valora en función de cómo crece la mayoría de los niños y adolescentes de una población de referencia representativa $(1,2)$. Una forma de comprobar el crecimiento adecuado de un individuo es mediante el uso de gráficas o curvas poblacionales. Las tablas de crecimiento utilizadas en la práctica clínica están 
divididas en centiles y, en el control individual de un niño, se aprecia cómo su crecimiento sigue de forma paralela un centil de la tabla (1). Este patrón es el considerado normal y, excepto en algunas variaciones fisiológicas, la desviación de ese centil por defecto o por exceso implica la posible existencia de un estado de malnutrición.

El crecimiento durante la lactancia o primera infancia, como extensión del patrón fetal, depende todavía de la relación entre el aporte de nutrientes y su incorporación a los tejidos mediante la insulina. Más tarde, el crecimiento infantil dependerá principalmente de la hormona del crecimiento (2). Además, como fenómeno individual, los niños con peso al nacer en los extremos del rango normal (retrasos del crecimiento o hipercrecimientos intrauterinos), pueden mostrar patrones de crecimiento fisiológico postnatal que se desvíen de los percentiles habituales buscando lo que será su carril de crecimiento durante la infancia (2).

\section{Tablas y gráficas de crecimiento}

La elaboración de las tablas de crecimiento se suele realizar a partir de diferentes tipos de muestreo (1):

a) Muestreo transversal en un momento determinado, es decir, medir los niños de diferentes edades al mismo tiempo.

b) Un muestreo longitudinal que consiste en seguir una cohorte de niños mientras crecen.

c) Un muestreo mixto en el que, sobre un estudio longitudinal, se van incorporando datos transversales. 
En este sentido, el muestreo longitudinal es el más útil cuando el clínico quiere estudiar el crecimiento individual y las diferentes variantes de la normalidad porque los datos se han obtenido a partir de un mismo grupo de niños en crecimiento. Por otro lado, como inconvenientes de los muestreos longitudinales cabe señalar que suelen contemplar un número reducido de participantes en la muestra seleccionada, se pierden niños durante los años que dura el estudio $\mathrm{y}$ las curvas pueden no estar actualizadas epidemiológicamente si han existido fenómenos de aceleración secular del crecimiento, especialmente cuando los datos registrados son de niños que nacieron 20 ó 25 años atrás. Todos estos inconvenientes se pueden minimizar con la elaboración de tablas a partir de muestreos de tipo mixto (1). Otro dato a tener en cuenta, sobre todo en nuestro medio, es el importante cambio poblacional que ha supuesto la llegada de gran número de inmigrantes y que, en la actualidad, contribuyen a un 17-20\% de los nacimientos (8), cifra que se ha multiplicado por 6 en la última década. Este fenómeno social conlleva una rápida modificación étnica y cultural de la población, aportando factores moduladores del crecimiento y de las pautas de alimentación de los niños que actualmente se controlan en los servicios de Atención Primaria.

En la actualidad se dispone de una serie de gráficas y tablas, tanto nacionales como internacionales, que se utilizan como estándares para la monitorización del crecimiento:

1) Las publicadas por la OMS (9) y elaboradas a partir de un estudio longitudinal en 1737 niños y niñas, alimentados con lactancia materna exclusiva, nacidos en Ghana, Brasil, India, Noruega, Omán y Estados Unidos de Norteamérica; durante el periodo 1997-2003. 
2) Las confeccionadas en el estudio longitudinal Euro-Growth (10), con los datos de 2245 niños y niñas, nacidos durante el periodo 1990-1996 en diferentes países europeos: España (que aportó una cuarta parte del total de la muestra), Austria, Alemania, Francia, Grecia, Reino Unido, Hungría, Italia, Irlanda, Croacia, Portugal y Suecia.

3) Las de Hernández-Sobradillo et al. (Fundación Orbegozo) (11, 12), a partir de un estudio longitudinal en 600 niños y niñas nacidos en el área metropolitana de Bilbao durante el periodo 1978-1980; y otro más reciente transversal en 6443 niños y niñas de la misma ubicación geográfica durante 2000-2001, con edades entre 0 y 18 años.

4) Las de Ferrández et al. (Fundación Andrea Prader) (13), elaboradas longitudinalmente con 332 niños y niñas nacidos en el área metropolitana de Zaragoza durante el periodo 1980-1986.

5) Las de Carrascosa et al. 2003 (14), también longitudinales, con los datos de 511 niños y niñas del área metropolitana de Barcelona nacidos durante el periodo 1998-2000.

6) Las de Carrascosa et al. 2008 (15), que son la fusión de las dos anteriores (Ferrández et al. y Carrascosa et al. 2003).

Entre las gráficas de crecimiento existe una serie de diferencias que se hacen más patentes en determinadas edades, pudiendo influir en que un valor antropométrico sea interpretado como normal (valores entre los percentiles 3 y 97) o anormal en dependencia de los estándares seleccionados. Un ejemplo concreto podría ser el valor que figura para el percentil 3 del peso en niñas en las gráficas de la OMS a los 12 meses (7100 g) en comparación con el del Euro-Growth que es aproximadamente un $10 \%$ mayor (7780 g). 


\section{Alimentación del lactante. Lactancia materna}

Respecto a la alimentación del lactante, también existe una serie de aspectos de gran interés; entre otros: el estudio de la prevalencia de lactancia materna (LM), los factores que determinan el tipo de lactancia y el abandono de la LM, el momento de inicio y la modalidad de introducción progresiva de la alimentación complementaria, y los patrones de crecimiento según el tipo de alimentación recibida. La LM es el alimento ideal y exclusivo que se recomienda durante los primeros 6 meses de la vida $\mathrm{y}$, a partir de ese momento, en compañía de otros alimentos o de alimentación complementaria (16).

Respecto al momento de finalización de la LM, si no hay inconveniente y la dieta es adecuada globalmente, la OMS y UNICEF recomiendan mantenerla hasta los 2 años o hasta que el niño y la madre quieran (16). En esta misma línea, la Academia Americana de Pediatría aconseja continuar con la LM al menos durante todo el primer año de la vida. Paradójicamente, el tiempo medio de mantenimiento de la LM no es muy alto en nuestro medio ( $<3-5$ meses) pese al buen conocimiento de sus beneficios para la salud, la información administrada durante la preparación al parto, y los consejos y medidas de promoción de la LM instauradas y divulgadas en los hospitales o desde los grupos de apoyo a la lactancia (17-25). El abandono precoz de la LM (hasta $1 / 3$ de los casos en el primer mes) refleja las dificultades sociales, culturales y educacionales que impiden su instauración y mantenimiento prolongado. Otro momento crítico de abandono de la LM son los 3-6 meses, coincidiendo con la reincorporación de la madre al trabajo y la inadecuada interpretación de ciertos cambios en los hábitos de alimentación que experimenta el propio bebé (21-25). En 
España, a los 3 meses de vida solo un 50\% de los niños toma LM y a los 6 meses un 10-20\%, o incluso menos, la sigue manteniendo (21-25). En estudios realizados recientemente en Aragón (Huesca y Alcañiz) (22, 23), los motivos más importantes que alegaron para dejar de lactar fueron la hipogalactia, sensación de hambre por parte del niño, decisión familiar y causas laborales o introducción de la alimentación complementaria.

Los beneficios para la salud que confiere la LM, tanto a corto como a largo plazo, han sido ampliamente contrastados en la literatura médica (16, 17, 26, 27), y quedan sintetizados en los siguientes puntos:

a) Crecimiento, desarrollo y maduración óptimos del recién nacido y el lactante.

b) Adecuación nutricional y biológica de la leche humana en cada momento evolutivo, con modificaciones en su composición según los requerimientos.

c) Función óptima fisiológica tanto digestiva como metabólica.

d) Prevención de enfermedades o alteraciones que pueden aparecer en el neonato y el lactante: alergia, estreñimiento, ciertas afecciones del tracto intestinal como los cólicos del lactante.

e) Influencia sobre enfermedades crónicas típicas del adulto que pueden originarse o «programarse» ya en las primeras etapas de la vida: alergia, enfermedades autoinmunes, obesidad, síndrome metabólico (dislipemias, hipertensión arterial, hiperinsulinismo, alteración del metabolismo hidrocarbonado).

f) Fomento del vínculo físico y emocional entre la madre y el recién nacido, manteniendo el que ya existía dentro del útero. 
Uno de los beneficios de la LM de gran interés en la actualidad es la disminución de la prevalencia de obesidad en etapas posteriores de la vida. El tiempo de amamantamiento se relaciona inversamente con el riesgo de aparición de sobrepeso y obesidad en niños y adolescentes; cuanto más prolongada ha sido la LM mayor es el efecto protector frente a la obesidad a largo plazo $(7,27)$. Los factores que explican las asociaciones entre la alimentación del lactante y la variabilidad de su patrón de crecimiento y adiposidad en etapas posteriores de la vida también se relacionan con la hipótesis de la programación metabólica inducida por la cantidad de energía y la composición nutricional de los alimentos ingeridos al inicio de la vida (sobre todo su componente proteico) (7). El periodo de la lactancia y de la introducción de la alimentación complementaria constituye una etapa muy sensible e importante de la vida del niño, siendo un momento crítico para la promoción óptima del crecimiento, la salud y el desarrollo.

\section{Justificación}

Por el momento, ni en nuestro medio ni en el resto de las Comunidades Autónomas existen datos epidemiológicos completos y actualizados acerca de los siguientes aspectos:

a) Patrón de crecimiento postnatal precoz y tardío de los niños menores de 24 meses, incluyendo el compartimento graso.

b) Cuáles son los estándares y curvas poblacionales que mejor se adaptan globalmente a los patrones de crecimiento en estas edades y, sobre todo, en subgrupos con riesgo nutricional o con alguna característica específica que pueda interferir en el crecimiento. 
c) Epidemiología de la alimentación con LM y de los hábitos alimenticios en niños menores de 24 meses.

d) La influencia sobre todo lo anterior de los factores de riesgo socioculturales y sanitarios, o los cambios que han podido ocasionar la llegada masiva de grupos de inmigrantes, etc.

Los profesionales de la salud, gracias a su esfuerzo y experiencia, abordan este tipo de cuestiones tan necesarias para su actividad diaria pero sin el apoyo de resultados contrastados científicamente. Así pues, se necesitan trabajos controlados y bien diseñados que evalúen longitudinalmente todos los aspectos mencionados, en una muestra significativa y representativa de niños, y cuyos resultados sirvan para saber cuál es la situación actual en nuestro medio. De esta manera, el conocimiento de los factores que influyen en la variabilidad del crecimiento y en el patrón alimentario en menores de 24 meses de edad, así como la detección de grupos de riesgo nutricional durante esta época tan vulnerable de la vida, ayudarán a mejorar aspectos relacionados con el manejo del niño sano en Atención Primaria y en la planificación de programas preventivos de intervención dirigidos a los grupos de riesgo. Un crecimiento óptimo durante los primeros meses de la vida aportará beneficios, a corto y largo plazo, disminuyendo el riesgo de alteraciones que aparecen en etapas posteriores inducidas por aspectos nutricionales que ocurrieron al inicio de la vida. 


\subsection{OBJETIVOS}

\section{Objetivo principal}

Valorar el patrón de crecimiento actual, la composición corporal y las pautas de alimentación de una muestra representativa de niños aragoneses hasta los 24 meses de edad; así como los factores prenatales, postnatales y psico-sociales que puedan influenciarlos.

\section{Objetivos secundarios}

1. Comprobar cuáles son los estándares poblacionales de referencia y las curvas de crecimiento ya existentes, tanto nacionales como internacionales, que mejor se adaptan al patrón actual de crecimiento de los niños aragoneses hasta los 24 meses de edad.

2. Registrar la prevalencia de lactancia materna en Aragón, así como los factores que intervienen en su instauración, mantenimiento y abandono.

3. Estudiar el crecimiento postnatal y la composición corporal de los menores de 24 meses de edad en función del tipo de lactancia y alimentación complementaria recibida durante los primeros meses de la vida.

4. Obtener datos epidemiológicos globales sobre el crecimiento, composición corporal y alimentación de los niños aragoneses menores de 24 meses que sirvan para conocer la situación actual, considerando aspectos demográficos, sociales y étnicos; y que, posteriormente, puedan ser utilizados como punto de partida en futuros proyectos de intervención y prevención dirigidos a determinados grupos de riesgo. 


\subsection{METODOLOGÍA}

\section{Diseño}

Se trata de un estudio observacional longitudinal en una cohorte representativa de la población de niños aragoneses desde el nacimiento hasta los 24 meses de vida.

\section{Sujetos de estudio}

En primer lugar, se realiza un muestreo entre los Centros de Salud (CS) representativos de la Comunidad Autónoma de Aragón que cumplan los siguientes criterios de inclusión: estar dotados de personal de pediatría y enfermería que realicen el programa de seguimiento del niño sano, con al menos dos años de antigüedad, con cumplimiento y cobertura de dicho programa superior al $80 \%$ de la población asignada. El cumplimiento y cobertura del programa de seguimiento del niño sano en los CS de Aragón es del $90 \%$ en los menores de 24 meses. Los sujetos que se incluirán en el estudio serán todos los nacidos durante el año 2009 (o un año natural si no se comienza en enero), que acudan desde la primera visita a las revisiones programadas por los cupos de Atención Primaria de los CS seleccionados, y cuyos padres hayan firmado el consentimiento informado. Se excluirán los niños que presenten malformaciones, enfermedades o condicionamientos y minusvalías físicas que provoquen alteraciones del crecimiento y/o del estado nutricional; así como los niños que se incorporen a los cupos de Atención Primaria tras el primer mes de vida por traslado o inasistencia. 


\section{Tamaño muestral}

Para establecer el tamaño de la muestra se han considerado los siguientes datos:

- Resultados sobre el tamaño y distribución de los nacidos en Aragón durante el último año publicado (12326 nacidos en 2006; Zaragoza 76 $\%$, Huesca $15 \%$ y Teruel $9 \%$; consultado en las bases del INE en marzo de 2008) y su tendencia creciente en los últimos años que podría alcanzar, en 2009, los 14000 nacidos aproximadamente.

- La varianza del peso, como variable antropométrica que mejor se relaciona con el patrón de crecimiento durante los primeros meses de la vida, estimado con un nivel de seguridad del $95 \%$ (error alfa $=$ $0,05)$ y un error máximo de medida de +/- 50 gramos. Las medias y desviaciones estándar utilizadas son las publicadas para la población aragonesa menor de 2 años (13).

A partir de los datos anteriores y considerando que un $10 \%$ de los niños no participará o se perderá durante el estudio, el número definitivo de niños necesarios será de 1540 (1170 en Zaragoza, 231 en Huesca y 139 en Teruel). Este es el número teórico de niños a tener en cuenta para que el muestreo aleatorio de los CS en cada provincia asegure el tamaño muestral. Una participación superior permitirá una mayor potencia estadística. 


\subsection{VARIABLES CONTEMPLADAS INICIALMENTE}

\section{Variables}

Las variables se recogerán en cada niño por el propio personal de enfermería y de pediatría de los CS seleccionados, en las visitas programadas para el seguimiento del niño sano en Atención Primaria (a los 15 días de vida, y en los meses $1^{\circ}, 2^{\circ}, 3^{\circ}, 4^{\circ}, 6^{\circ}, 9^{\circ}, 12^{\circ}, 15^{\circ}, 18^{\circ}$ y $24^{\circ}$ de vida). Las variables se medirán, estimarán y registrarán siguiendo la metodología estándar y consensuada. De esta manera, en una primera fase, el personal de pediatría y enfermería realizará un curso de adiestramiento que recibirá de un Grupo de Asesoramiento formado por miembros del propio grupo investigador del presente proyecto. La formación inicial tendrá entre los contenidos teóricos y prácticos la obtención de datos demográficos, obstétricos, perinatales y de la valoración nutricional del niño. Durante este periodo de formación también se determinará el nivel de conocimientos del personal sanitario sobre alimentación y crecimiento infantil y se realizarán talleres donde se comprobará la técnica y la variabilidad inter e intra observador. En cada uno de los niños se determinarán las siguientes variables:

- Datos demográficos: Fecha de nacimiento, sexo, país de origen de los padres, localidad donde habita, número de hermanos y orden en la fratría, trabajo y nivel de estudios de los padres.

- Historia clínica obstétrica y perinatal: Antropometría materna antes y al finalizar la gestación, antropometría paterna, existencia de buen control gestacional, incidencias gestacionales, hábito tabáquico de la madre durante el embarazo, edad gestacional al nacer, tipo de parto, 
incidencias neonatales, antecedentes maternos o del niño que contraindiquen o dificulten la LM, antropometría del recién nacido, días de estancia hospitalaria tras el parto, peso del niño al alta hospitalaria tras el parto, alimentación del niño tras el parto, días de vida en la primera consulta en CS.

- Alimentación: Duración de la lactancia materna exclusiva, momento del destete definitivo y motivo de abandono de la LM, momento de comienzo de la leche de fórmula y tipo, solicitud o administración institucional de apoyo para la LM, utilización de métodos de extracción y conservación de la LM (momento y motivo), pauta de introducción de la alimentación complementaria, asistencia a comedor en la guardería.

- Antropometría: Peso $(\mathrm{kg})$, longitud $(\mathrm{cm})$, perímetro cefálico $(\mathrm{cm})$, perímetro abdominal $(\mathrm{cm})$, perímetro braquial $(\mathrm{cm})$, pliegues cutáneos (mm) con lipómetro de compás.

\subsection{RECOGIDA Y ANÁLISIS DE DATOS}

El registro de las variables se cumplimentará en los cuestionarios confeccionados específicamente para el estudio. Se diseñará ad hoc una base de datos en Excel (Microsoft) en la que se archivarán todos los datos recogidos y los datos identificativos de los participantes que se protegerán y encriptarán. 
El análisis estadístico se realizará mediante el paquete estadístico SPSS. En primer lugar, se llevará a cabo la estadística descriptiva univariada. Las variables cualitativas se presentarán mediante la distribución de frecuencias de los porcentajes de cada categoría. Para las variables cuantitativas se explorará si siguen o no una distribución normal mediante la prueba de Kolmogorov-Smirnov, y se darán indicadores de tendencia central (media o mediana) y de dispersión (desviación estándar o percentiles).

En la fase de estadística analítica, se realizará análisis bivariado y multivariado para investigar los factores prenatales, postnatales y psicosociales relacionados con la variabilidad del crecimiento y la alimentación en este grupo poblacional. La asociación entre estos factores se investigará mediante pruebas de contraste de hipótesis, con comparación de proporciones cuando ambas sean cualitativas (chi cuadrado, prueba exacta de Fisher); comparaciones de medias cuando una de ellas sea cuantitativa ( $t$ de Student, ANOVA, y si no siguen distribución normal el test de la U de Mann-Whitney o el de Kruskall-Wallis); y con pruebas de regresión lineal cuando la variable dependiente sea cuantitativa. Adicionalmente se construirán modelos multivariantes de regresión logística y regresión lineal múltiple que permitirán determinar cuáles han sido los factores relacionados con los patrones de crecimiento y de alimentación. El análisis será complementado con representaciones gráficas. El nivel de significación estadística para este estudio será $\mathrm{p}<0,05$. 


\subsection{ASPECTOS ÉTICOS}

El estudio se llevará a cabo siguiendo las normas deontológicas reconocidas por la Declaración de Helsinki (52. a Asamblea General de Edimburgo, Escocia, octubre de 2000), las Normas de Buena Práctica Clínica y cumpliendo la legislación vigente y la normativa legal vigente española que regula la investigación clínica en humanos (Real Decreto 223/2004 sobre ensayos clínicos y Ley 14/2007 de Investigación Biomédica). Antes del comienzo del estudio, se explicará detalladamente el estudio que se pretende realizar y se pedirá conformidad previa por escrito por parte de cada individuo y de su padre/madre o tutor/a. Los datos serán protegidos de usos no permitidos por personas ajenas a la investigación y se respetará la confidencialidad de los mismos de acuerdo a la Ley Orgánica 15/1999, de 13 de diciembre, sobre la Protección de Datos de Carácter Personal y la ley 41/2002, de 14 de noviembre, ley básica reguladora de la autonomía del paciente $\mathrm{y}$ de derechos $\mathrm{y}$ obligaciones en materia de información y documentación clínica. Por tanto, la información generada en este ensayo será considerada estrictamente confidencial, entre las partes participantes, permitiéndose, sin embargo, su inspección por las Autoridades Sanitarias.

\subsection{LIMITACIONES DEL ESTUDIO}

Las limitaciones de este estudio, como en otros observacionales de tipo longitudinal, se fundamentan en los posibles sesgos que se intentarán minimizar en la mayor medida. 
En primer lugar, la selección de la muestra se realizará entre los Centros de Salud de Aragón (unidad de muestreo), teniendo en cuenta que la población contemplada represente a la de los niños nacidos durante el año 2009 en cada provincia y, a su vez, considere la contribución proporcional de cada provincia al total de nacidos en Aragón, tanto en número como en ratio de población rural/urbana. En comparación con un muestreo aleatorio entre la población total de niños, el muestreo por centros minimiza los sesgos más importantes del estudio durante los 24 meses de seguimiento, mejorando la fiabilidad del registro de las variables al optimizar el adiestramiento y eficacia del binomio pediatría-enfermería. De otra manera, casi todos los equipos de pediatría-enfermería podrían estar reclutados, si aleatoriamente se hubiera seleccionado algún niño de su cupo de Atención Primaria, y entonces las diferencias inter-observador aumentarían, perdiendo precisión y exactitud en el registro y control de las variables. Además, al considerar a todos los niños que acuden a un centro no se creará sensación de discriminación o de falta de atención entre los familiares de los niños que resultaran no incluidos. El tamaño de la muestra de niños que nacerán en 2009 es una estimación aproximada a partir de los últimos datos publicados del INE (correspondiente a 2006, consultados en marzo de 2008) y su tendencia durante los últimos años; se cuenta además con un margen de seguridad de un $10 \%$ considerando los niños que no participen o que se pierdan durante el estudio. Conviene añadir que existen también limitaciones en relación con los propios CS, principalmente si alguno de los pediatras no quiere participar o cambia su destino laboral durante el periodo considerado.

Otros sesgos que pueden aparecer son los provocados por las influencias externas sociales, personales y factores ambientales a lo largo del seguimiento. En el estudio longitudinal se deberán controlar y registrar todos 
los factores que puedan interferir en el patrón de crecimiento y las pautas de alimentación durante los 24 meses de seguimiento. Un reto importante será el de tipo logístico, para el adiestramiento de los profesionales, el reclutamiento de los niños y el seguimiento de una muestra tan amplia de niños y familiares. El esfuerzo para la coordinación del mismo implicará tiempo y dedicación. Un punto favorable del presente estudio es que se adapta perfectamente al formato del programa de seguimiento del niño sano que habitualmente se realiza en los centros de Atención Primaria.

Respecto a la medición y registro de las variables, sobre todo las antropométricas, pueden estar sujetas a variabilidad. Con el fin de disminuir la variabilidad intra e interobservador, se realizarán previamente talleres prácticos de entrenamiento técnico destinados al personal participante en el estudio, se registrará dicha variabilidad para conocerla antes del inicio, y solo se considerarán los resultados obtenidos por las personas adiestradas para la ocasión.

\subsection{CRONOGRAMA Y DISTRIBUCIÓN DE LAS TAREAS}

El profesor José L. Olivares López, Catedrático de Pediatría, es el Investigador Principal y responsable directo del diseño, dirección y coordinación científica del proyecto. El grupo CORE (ver apartado 6.1) con varios de sus componentes detallados a continuación se encargará de las tareas concretas relacionadas con el diseño y coordinación del proyecto CALINA. 
Preparación y planificación. Duración: 3 meses, del mes $1 .^{\circ}$ al 3. ${ }^{\circ}$.

Luis A. Moreno Aznar, Gerardo Rodríguez Martínez y M.a Pilar Samper Villagrasa. Profesores de la Universidad de Zaragoza (Área de Pediatría). Responsables del diseño científico del proyecto, así como de la planificación, coordinación y desarrollo de los puntos incluidos en esta etapa. José M. Mengual Gil, Pediatra, Director del Área de Transferencia del Conocimiento del Instituto Aragonés de Ciencias de la Salud es el responsable de la logística, gestión de recursos y reclutamiento de personal sanitario colaborador.

Tareas de la preparación y planificación:

- Selección de los centros participantes y del personal colaborador.

- Perfeccionamiento del diseño, búsqueda de documentación y fuentes bibliográficas.

- Reuniones científicas para unificar criterios, ultimar detalles y estandarizar la metodología.

- Elaboración de la base de datos, documentos para la recogida de datos y cuestionarios.

- Adquisición de bienes necesarios.

○ Formación teórica y adiestramiento práctico del personal mediante talleres.

- Estudio piloto, valoración de la variabilidad inter e intraobservador. 
Desarrollo del estudio longitudinal. Duración: 30 meses, del mes $4 .^{\circ}$ al $33 .^{\circ}$.

Los pediatras de Atención Primaria, especialmente los miembros del grupo CORE (ver apartado 6.1.), serán los responsables de la coordinación del trabajo de campo en los centros de Atención Primaria, seguimiento del proyecto, organización asistencial del personal sanitario y registro de las variables.

Tareas durante el estudio longitudinal

- Información a la familia de los objetivos y características del estudio. Solicitud de su colaboración y del consentimiento informado. Comprobación de si cumplen los criterios de inclusión/exclusión. Reclutamiento.

- Registro de los datos demográficos e historia obstétrica y perinatal. Determinación y registro longitudinal de las variables (crecimiento y alimentación) en los niños seleccionados durante el año 2009 (o año natural completo si no comienza en enero) en cada uno de los momentos previstos hasta los 24 meses de edad.

Análisis y difusión de resultados. Duración de 7 meses, del mes $30 .^{\circ}$ al $36 .^{\circ}$ (Solapada con la anterior).

Las tareas de análisis y difusión de los resultados estarán coordinadas por los siguientes investigadores: José L. Olivares López, Luis A. Moreno Aznar y Gerardo Rodríguez Martínez; participando el resto del equipo investigador en la evaluación, interpretación y difusión de los mismos. 
Tareas de análisis y difusión de resultados

- Realización del análisis estadístico. Evaluación e interpretación de resultados (los relativos a ciertos aspectos del $1 .^{\text {er }}$ año de la vida se podrán obtener preliminarmente antes de la finalización de la fase 2).

- Elaboración del informe final, presentación y difusión de resultados definitivos.

Prediseño de subestudios y otras acciones que continúen y consoliden la presente línea de investigación.

\subsection{BIBLIOGRAFÍA}

1. Sarría A, Bueno M, Rodríguez G. Exploración del estado nutricional. En: Bueno M, Sarría A, Pérez-González JM, eds. Nutrición en Pediatría. Ergon, Madrid 2007; p. 27-41.

2. Tojo R, Leis R. Crecimiento normal. En: Cruz M, ed. Tratado de Pediatría. Ergon, Madrid 2006; p. 845-856.

3. Carrascosa A, Ballabriga A. Crecimiento intrauterino. En: Argente J, et al. eds.Tratado de Endocrinología pediátrica y de la adolescencia. Doyma, Barcelona 2000; p. 1-3.

4. Dulloo AG. Thrifty energy metabolism in catch-up growth trajectories to insulin and leptin resistance. Best Pract Res Clin Endocrinol Metab 2008; 22: $155-171$.

5. Demmelmair H, von Rosen J, Koletzko B. Long-term consequences of early nutrition. Early Hum Dev 2006; 82: 567-574. 
6. Reilly JJ, Armstrong J, Dorosty AR, et al. Early life risk factors for obesity in childhood: cohort study. BMJ 2005; 330: 1357.

7. Moreno LA, Rodríguez G. Dietary risk factors for development of childhood obesity. Curr Op Clin Nutr Metab Care 2007; 10: 336-341.

8. Rodríguez G, Olivares JL, Fleta J, Moreno LA. Estado nutricional de los niños inmigrantes, adoptados y refugiados. En: Bueno M, Sarría A, Pérez JM, eds. Nutrición en Pediatría. Ergon, Madrid 2007; p. 525-534.

9. WHO Multicentre Growth Reference Study Group. WHO Child Growth Standards: length/height-for-age, weight-for-age, weight-for-length, weight-for-height and body mass index-for-age: methods and development. Geneva: World Health Organization, 2006.

10. Haschke F, Van't Hof MA. Euro-Growth references for length, weight, and body circumferences. J Pediatr Gastroenterol Nutr 2000; 31Suppl 1: S14-S38.

11. Hernández M, Castellet J, Narvaíza JL, et al. Curvas y tablas de crecimiento. Estudio semi-longitudinal. Fundación F. Orbegozo. Bilbao 1988. En: Carrascosa A, et al. eds. Patrones de crecimiento y desarrollo en España. Ergon, Madrid 2004; p. 117-143.

12. Sobradillo B, Aguirre A, Aresti U, et al. Curvas y tablas de crecimiento. Estudios longitudinal y transversal. Fundación F. Orbegozo. Bilbao 2002. En: Carrascosa A, et al. eds. Patrones de crecimiento y desarrollo en España. Ergon, Madrid 2004; p. 145-168.

13. Ferrández A, Mayayo E, Labarta JI, et al. Estudio longitudinal de crecimiento y desarrollo. Centro Andrea Prader. Zaragoza 1980-2002. En: Carrascosa A, et al. eds. Patrones de crecimiento y desarrollo en España. Ergon, Madrid 2004; p. 61-116. 
14. Carrascosa A, Copil A, Yeste D, Gussinyé M. Patrones de crecimiento en niños normales tras el nacimiento y hasta la edad adulta. Barcelona 2003. En: Carrascosa A, et al. eds. Patrones de crecimiento y desarrollo en España. Ergon, Madrid 2004; p. 49-60.

15. Carrascosa A, Fernández JM, Fernández C, Ferrández A, López-Siguero JP, Sánchez E, Sobradillo B, Yeste D y grupo colaborador español. Estudio transversal español de crecimiento 2008. Parte II: valores de talla, peso e índice de masa corporal desde el nacimiento a la talla adulta. An Pediatr 2008; 68: 552-569.

16. OMS. Nutrición del lactante y del niño pequeño. Estrategia mundial para la alimentación del lactante y del niño pequeño. Informe de la Secretaría. 55 Asamblea Mundial de la Salud, 16 de abril de 2002.

17. Lactancia materna: guía para profesionales. Comité de la Lactancia Materna de la AEP. Monografías de la AEP n. ${ }^{\circ}$ 5. Madrid: Ergon, 2004.

18. Hernández MT, Aguayo J. La lactancia materna. Cómo promover y apoyar la lactancia materna en la práctica pediátrica. Recomendaciones del Comité de la Lactancia AEP. An Pediatr (Barc) 2005; 63: 340-356.

19. Hostalog AM, Sorní A, Jovaní L, et al. Lactancia materna en el sur de Cataluña. Estudio de factores socioculturales y sanitarios que influyen en su elección y mantenimiento. An Esp Pediatr 2001; 54: 297-302.

20. Jovani L, Gutiérrez P, Aguilar C, et al. Influencia del personal sanitario de asistencia primaria en la prevalencia de la lactancia materna. An Esp Pediatr 2002; 57: 534-539.

21. Estévez MD, Martell D, Medina R, et al. Factores relacionados con el abandono de la lactancia materna. An Esp Pediatr 2002; 56: 144-150.

22. Guerrero C, Garafulla J, Lozano D, et al. Estudio lactancia en área hospital de Alcañiz. ¿Merece la pena poner en práctica los diez pasos de 
IHAN en un hospital comarcal? Bol Pediatr Arag Rioj Sor 2006; 36: 2026.

23. Rodríguez G, Fuertes J, Samper MP, Broto P, Collado MP, Sebastián MF, Pardos C, Solanas AB. Programas de intervención para promocionar la lactancia materna. Proyecto PALMA. Acta Pediatr Esp 2008; 66: 564568.

24. González M, Toledano J. La lactancia materna en nuestro medio: análisis de la situación. Acta Pediatr Esp 2007; 65: 123-125.

25. Suárez P, Alonso J, López A, et al. Prevalencia y duración de la lactancia materna en Asturias. Gac Sanit 2001; 15: 104-110.

26. Schack-Nielsen L, Michaelsen KF. Breast feeding and future health. Curr Opin Clin Nutr Metab Care 2006; 9: 289-296.

27. Arenz S, Ruckerl R, Koletzko B, von Kries R. Breast-feeding and childhood obesity. A systematic review. Int J Obes Relat Metab Disord 2004; $28: 1247-1256$. 


\section{EXPERIENCIA DEL EQUIPO INVESTIGADOR SOBRE EL TEMA}

El equipo de investigación GENUD (Growth, Exercise, NUtrition and Development), se ha interesado desde su creación en el estudio de los estilos de vida y los patrones de alimentación, y su relación con el crecimiento y desarrollo en niños de todas las edades. Aunque el Grupo GENUD está acreditado por el Gobierno de Aragón como Grupo Consolidado desde 2002 (BOA de 18 de Diciembre) y como Grupo de Excelencia desde 2008 (BOA de 9 de Mayo), sus componentes más expertos ya tenían previamente una trayectoria sólida en relación con el estudio nutricional y el crecimiento en pediatría. El investigador responsable del equipo GENUD ha sido el profesor don Manuel Bueno Sánchez y, desde 2008, el profesor don Luis A. Moreno Aznar ha cogido el relevo. Las líneas de investigación más importantes de los últimos años vienen resumidas en los diferentes proyectos financiados y que han proporcionado resultados muy interesantes, contribuyendo a que el grupo sea considerado en su área como referente tanto nacional como internacional.

\section{Líneas de investigación}

- Ingesta y gasto energéticos como factores de riesgo de aparición de obesidad en niñas (FIS 97/0531; 1997-99). Objetivos: Valoración conjunta de los factores de riesgo de sobrepeso/obesidad y sus determinantes en un grupo de niñas obesas: energía ingerida, cantidad y proporción de nutrientes, gasto energético en reposo, porcentaje de 
substratos oxidados y actividad física. Investigador Principal (IP): Manuel Bueno Sánchez.

- Proyecto AVENA. Los adolescentes españoles ante la encrucijada nutricional del próximo milenio. Valoración de su estado nutricional y de sus hábitos alimentarios, de ocio y de comportamiento. Propuestas de intervención (FIS 00/0015; 2000-2002). Objetivos: Evaluación de la situación nutricional y metabólica en adolescentes españoles. Obtención de datos de actividad y condición física, ingesta dietética habitual y composición corporal, representativos de la población adolescente española. IP: Ascensión Marcos Sánchez.

- Proyecto HELENA. Healthy lifestyle in Europe by nutrition in adolescence. (Unión Europea, 6. ${ }^{\circ}$ Programa Marco. Contrato FOODCT-2005-007034; 2005-2008). Objetivos: Desarrollar y armonizar métodos innovadores para la evaluación de los hábitos de vida de los adolescentes europeos, y utilizarlos concretamente en la valoración de la dieta, la nutrición y la actividad física. IP: Luis A. Moreno Aznar.

- Proyecto EVASYON. Desarrollo, aplicación y evaluación de la eficacia de un programa terapeútico para adolescentes con sobrepeso y obesidad: educación integral nutricional y de actividad física (FIS PI05/1579; 2005-2008). Objetivos: Desarrollar entre un grupo multidisciplinar de profesionales de la salud un programa terapéutico de intervención nutricional, incluyendo aspectos dietéticos y de actividad física, para disminuir la prevalencia de sobrepeso y obesidad en adolescentes españoles. IP: Zaragoza: Jesús M. ${ }^{a}$ Garagorri Otero.

- Proyecto IDEFICS. Identification and Prevention of Dietary and lifestyle induced health effects in children and infants. (Unión 
Europea, 6. ${ }^{\circ}$ Programa Marco. Contrato FOOD-CT-2006-016181-2; 2007-2011). Objetivos: Prevención primaria de la obesidad en escolares prepuberales (2-10 años) mediante una intervención de tipo multicéntrico en 11 países europeos y valoración de la efectividad de dicho programa global. IP: Luis A. Moreno Aznar.

- Proyecto EURRECA. Red de Excelencia denominada «European Recommendations Aligned»(Unión Europea, 6. Programa Marco. FOOD-CT-2007-036196-2; 2007-2011). Objetivos: Establecer las recomendaciones de ingesta de micronutrientes para la población infantil, definiciones consensuadas sobre alimentación infantil en el contexto europeo, recopilar y revisar críticamente la información disponible para definir las ingestas dietéticas recomendadas para niños y alcanzar un consenso sobre estrategias para calcular las ingestas dietéticas recomendadas. IP: Luis A. Moreno Aznar.

- Proyecto PIANO. Proyecto educacional de intervención sobre alimentación y actividad física en niños oscenses (FIS PI07/1234; 2008-2010). Objetivo: Evaluar la efectividad de una intervención basada en dieta, actividad física y factores medioambientales para reducir la prevalencia de sobrepeso y obesidad en los escolares de Educación Primaria planificado desde Atención Primaria. IP: Gerardo Rodríguez Martínez.

- Proyecto PALMA. Evaluación de la eficacia de un programa multidisciplinar para la promoción de la alimentación con lactancia materna desde los servicios de Atención Primaria. (FIS MD 07/00045; 2008). Objetivos: Evaluar la efectividad de una propuesta de intervención básica de apoyo a la lactancia materna, realizada por el 
grupo multidisciplinar de Atención Primaria (pediatras, enfermeras y matrona) en los momentos clave donde se produce más tasa de abandono, así como los factores pre y postnatales que intervienen en la instauración, mantenimiento y abandono de la lactancia materna (incluyendo los aspectos sociales, culturales, psicológicos, sanitarios, laborales y educacionales). IP: Gerardo Rodríguez Martínez. 


\section{RELEVANCIA DEL PROYECTO}

\section{Impacto clínico, asistencial y/o desarrollo tecnológico}

El desarrollo del presente proyecto conllevará una serie de beneficios sobre la población de niños menores de 24 meses, contribuyendo al mejor conocimiento de los factores que influyen en la variabilidad de su crecimiento y en el patrón alimentario, así como en la detección de grupos de riesgo nutricional durante esta época tan vulnerable de la vida. A nivel asistencial ayudarán a mejorar aspectos relacionados con el manejo del niño sano en Atención Primaria y en la planificación de programas preventivos de intervención dirigidos a los grupos de riesgo detectados. Un crecimiento óptimo durante los primeros meses de la vida aportará beneficios, a corto y largo plazo, disminuyendo el riesgo posterior de aparición de alteraciones en relación con aspectos nutricionales que ocurrieron al inicio de la vida. Desde el punto de vista tecnológico, la información y el adiestramiento que supone el desarrollo del presente proyecto para el propio personal de pediatría y enfermería de Atención Primaria, responsables de la salud de los niños, tendrá un impacto beneficioso en las correspondientes áreas sanitarias donde estén ubicados los centros de salud que participen. Así mismo, la mejora asistencial que se obtenga en el manejo de los lactantes y de los niños pequeños desde Atención Primaria, a partir de los resultados del estudio, se podrá hacer extensiva al resto de áreas. El material adquirido, los métodos empleados y los resultados obtenidos quedarán como parte integrante de la práctica asistencial en las revisiones del niño sano. 


\section{Impacto bibliométrico de las publicaciones}

El impacto bibliométrico de las publicaciones que surjan de este proyecto relacionado con la salud de los lactantes y niños pequeños será importante, con toda probabilidad, como lo ha sido el de otros proyectos que nuestro grupo ha desarrollado previamente. Los resultados darán respuesta a unas hipótesis y desarrollarán unos objetivos que tienen en cuenta aspectos y factores relacionados con la variabilidad del crecimiento y desarrollo infantil que permanecen todavía sin esclarecer. 


\section{MEDIOS DISPONIBLES Y PRESUPUESTO}

Para el desarrollo del proyecto CALINA se dispone de la infraestructura y del personal docente-investigador de las instituciones participantes, dotado este de amplia experiencia en el adiestramiento, formación y coordinación en lo referente al crecimiento, composición corporal, alimentación y desarrollo durante la lactancia y la infancia.

El Área de Pediatría de la Universidad de Zaragoza y el Grupo GENUD (Gobierno de Aragón) prestarán apoyo investigador logístico, administrativo y metodológico:

- Acceso a bases de datos documentales (Medline, Cochrane, Ovid, ProQuest e IME).

- Equipos informáticos, software (paquete estadístico SPSS), acceso a Internet y medios audiovisuales.

- Estructura física con despachos, aulas y biblioteca.

El proyecto cuenta también con el apoyo logístico y metodológico de la Red de Investigación en Atención Primaria (Instituto Aragonés de Ciencias de la Salud), acreditada por el Instituto Carlos III (FIS 06/018), y el asesoramiento científico de Rosa Magallón Botalla, Coordinadora del Grupo Aragonés de Investigación en Atención Primaria (GAIAP).

En los Centros de Salud de Atención Primaria (Servicio Aragonés de Salud), se dispone de infraestructura asistencial y tecnológica, con medios tanto materiales como humanos: 
- Personal de pediatría y de enfermería de los centros que proporcionan: interés investigador, sobrada experiencia asistencial y vinculación estrecha con el niño y su familia.

- Entorno físico adecuado y material habitual de las consultas de pediatría utilizado para las revisiones rutinarias del niño sano: básculas para pesar bebés, tallímetros, cintas métricas, ordenadores y programa OMI para el registro de las variables. 


\section{DOCUMENTOS INSTITUCIONALES. APROBACIÓN DEL PROYECTO}

\subsection{INFORME DE VIABILIDAD DEL PROYECTO}

El proyecto CALINA fue evaluado favorablemente y considerado viable por la Comisión de Investigación del Instituto Aragonés de Ciencias de la Salud (abril 2008).

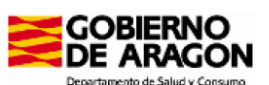

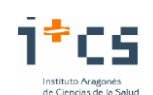

COMISION DE INVESTIGACION

\section{INFORME DE VIABILIDAD DE PROYECTOS DE INVESTIGACION}

El proyecto titulado: Crecimiento y alimentación durante la lactancia y la primera infancia en niños aragoneses (CALINA), cuyo investigador principal es José Luis Olivares López, ha sido presentado para su evaluación por parte de esta Comisión de Investigación que, una vez evaluado, considera que:

- La hipótesis y los objetivos son claros, concretos y con posibilidades de avance en el área de conocimiento en que se enmarca.

- La metodología es correcta, adecuada a los objetivos y bien planteada en sus distintos aspectos.

- La factibilidad es alta, dada la consistencia del grupo investigador, su trayectoria investigadora y su experiencia en esta línea.

- La relevancia científica y sanitaria prevista es importante.

Por todo ello, esta Comisión de Investigación considera que el proyecto es viable, 


\title{
5.2. COMITÉ ÉTICO DE INVESTIGACIÓN CLÍNICA DE ARAGÓN
}

\author{
El Comité Ético de Investigación Clínica de Aragón consideró como
} favorable el estudio CALINA, a fecha de 4 de junio de 2008.

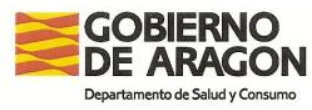

CEIC Aragón (CEICA)
Informe Dictamen Protocolo Favorable

C.P. ICS08/0088 - C.I. PI08/21 04 de junio de 2008

Dña. María González Hinjos, Secretaria del CEIC Aragón (CEICA)

CERTIFICA

10. Que este Comité en su reunión de 4 de junio de 2008, correspondiente al Acta $\mathrm{n}^{\circ} \mathrm{CP} 04 / 06 / 08$, ha evaluado la propuesta del investigador referida al estudio:

Título: Crecimiento y alimentación durante la lactancia y la primera infancia en niños aragoneses (calina)

Investigador: José Luis Olivares López

Versión Inicial Protocolo: abril 2008

Versión Inicial Hoja Información al Paciente: Versión 2 de 31/05/08

20. Considera que

- Se respetan los principios éticos básicos y es adecuado el procedimiento para obtener el consentimiento informado.

- Se realiza de conformidad con lo establecido en la Ley 14/2007 de Investigación Biomédica.

30. Por lo que este CEIC emite un DICTAMEN FAVORABLE.

Lo que firmo en Zaragoza, a 04 de junio de 2008

Fdo:

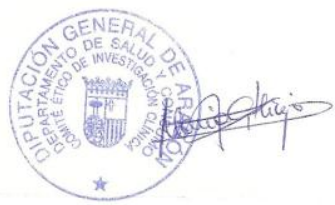

Dña. María González Hinjos Secretaria del CEIC Aragón (CEICA) 


\subsection{FINANCIACIÓN. INFORME DEL INSTITUTO DE SALUD CARLOS III}

En fecha de 27 de noviembre de 2008, se comunicó por parte del Instituto de Salud Carlos III (Subdirección General de Innovación y Fomento de la Investigación; Ministerio de Ciencia y Tecnología) la situación del proyecto CALINA como favorable, especificando la cuantía de presupuesto por anualidades y apartados. En dicho presupuesto se denegaba la financiación para personal contratado a cargo del proyecto.

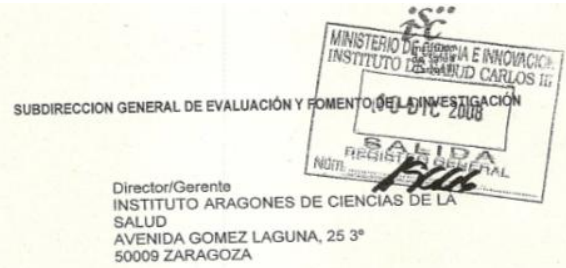

De acuerdo con lo establecido en el apartado 9 de la Resolución de 12 de marzo de 2008, del Instituto de Salud Carlos III, por la que se publica la marzo de 2008 , del instituto al an̂o 2008 de concesión de ayudas de la Acción Estratégica en Salud, en el marco del Plan Nacional de $1+D+12008$ 2011, como órgano instructor le notifico la:

"RESOLUCIÓN DE LA DIRECCIÓN DEL INSTITUTO DE SALUD CARLOS III POR LA QUE SE APRUEBA LA RELACIÓN DEFINITIVA DE RESULTADOS DEL SUBPROGRAMA DE SEROYECTOS DE INVESTIGACION EN SALUD, CORRESPONDIENTE A LA PROYETOS DORIA 2008, DE AYUDAS DE LA ACCIÓN ESTRATEGICA EN SALUD EN EL MARCO DEL PLAN NACIONAL DE I+D+1 2008-2011".

En consecuencia le comunico que la Dirección de este Instituto ha resuelto conceder a su centro los proyectos que se adjuntan, incluyendo sus condiciones económicas y composición del equipo investigador.

El plazo de ejecución de los proyectos y de presentación de memorias anuales y finales para su justificación se encuentra recogido en la resolución.

La cumplimentación de las memorias, cientificas y económicas, se realizará a través de la aplicación totongstiona fis iscilles (en caso de no disponer de contraseña de acceso a la misma deberá solicitarla por mail a la dirección gestiona fisaincili es).

Rogamos haga llegar esta información a los centros de realización de los proyectos.

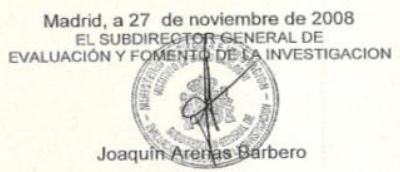




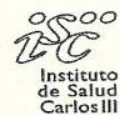

\section{MINISTERIO DE CIENCIA E INNOVACIÓN \\ SUBD IRECCIÓN GENERAL DE EVALUAC IÓN}

$Y$ FOMENTO DE LA INVESTIGACION

investigador Ppal.:

OLIVARES LOPEZ, JOSE LUIS

Expte: PI080559

Centro realización:

HOSPITAL CLINICO UNIVERSITARIO LOZANO BLESA

Centro solicitante:

INSTITUTO ARAGONES DE CIENCIAS DE LA SALUD

Titulo del proyecto:

CRECIMIENTO Y ALIMENTACIÓN DUFANTE LA

LACTANCIA Y LA PRIMERA INFANCIA EN

NIÑOS ARAGONESES (CALINA)

Duracion (años):

3

\begin{tabular}{|l|r|r|r|r|r|}
\hline ANUALIDAD & AÑO 1 & AÑO 2 & AÑO 3 & AÑ̃ 4 & Total \\
\hline \hline Personal & 0 & 0 & 0 & 0 & 0 \\
\hline Bienes y Servicios & 13.800 & 1.000 & 1.000 & 0 & 15.800 \\
\hline Viajes y Dietas & 800 & 600 & 800 & 0 & 2.200 \\
\hline Subtotal & 14.600 & $1.600,00$ & $1.800,00$ & 0,00 & $18.000,00$ \\
\hline $21 \%$ gastos generales & $3.066,00$ & 336,00 & 378,00 & 0,00 & $3.780,00$ \\
\hline TOTAL & $17.666,00$ & $1.936,00$ & $2.178,00$ & 0,00 & $21.780,00$ \\
\hline
\end{tabular}

Contratados concedidos:

Doctor:

Licenciado: 0

Diplomado: 0

Tecnico F.P.: 0 


\section{RECLUTAMIENTO DE CENTROS PARTICIPANTES EN EL PROYECTO. MUESTRA DEL PROYECTO CALINA}

\subsection{GRUPO CORE}

Como ya se ha comentado en el prefacio, la idea original del proyecto CALINA y el diseño preliminar del estudio partieron de un grupo multidisciplinar compuesto por miembros de la Universidad de Zaragoza, del Instituto Aragonés de Ciencias de la Salud y de pediatras de Atención Primaria de diferentes puntos de la Comunidad Autónoma Aragonesa.

Los miembros de este grupo pertenecientes a la Universidad de Zaragoza se encargaron de preparar la primera memoria, a partir del objetivo inicial de realizar un estudio global sobre el crecimiento de la población aragonesa de 0 a 24 meses y sobre los determinantes que podrían influir en el mismo. Este estudio ya estaba en mente de los diversos componentes del equipo investigador pero no se pudo materializar hasta que confluyó una serie de circunstancias que propiciaron los primeros contactos entre los miembros de la Universidad de Zaragoza, Instituto Aragonés de Ciencias de la Salud y los pediatras de Atención Primaria. Tras la primera reunión formal se constituyó el grupo CORE (término inglés que significa «núcleo»), con la intención de que fuera el núcleo coordinador del grupo de investigación definitivo y más amplio que englobaría a todos los participantes en el estudio CALINA.

En las reuniones de grupo CORE se perfiló el diseño preliminar del proyecto CALINA, se consensuaron los aspectos formales y metodológicos 
y, tras la aprobación del proyecto por el Instituto de Salud Carlos III, se trasladaron los objetivos del estudio CALINA a los diferentes centros de salud seleccionados. Los componentes del grupo CORE contribuyeron al reclutamiento del personal de dichos centros para formar el grupo de investigación definitivo.

\section{Componentes del Grupo CORE de Investigación CALINA} (Orden alfabético)

Jorge Fuertes Fernández-Espinar (C.S. Perpetuo Socorro, Huesca).

José Galbe Sánchez-Ventura (C.S. Torrero-La Paz, Zaragoza).

Nuria García Sánchez (C.S. Delicias Sur, Zaragoza).

Isabel Lostal Gracia (C.S. Actur Sur, Zaragoza).

Rosa Magallón Botalla (C.S. Arrabal, Zaragoza. Coordinadora del Grupo Aragonés de Investigación en Atención Primaria GAIAP).

José M. ${ }^{\mathrm{a}}$ Mengual Gil (Director del Área de Transferencia del Conocimiento. Instituto Aragonés de Ciencias de la Salud).

Isabel Moneo Hernández (C.S. Las Fuentes Norte, Zaragoza).

Luis A. Moreno Aznar (Universidad de Zaragoza).

Elena Muñoz Jalle (H.C.U. Lozano Blesa).

José Luis Olivares López (Universidad de Zaragoza).

Gerardo Rodríguez Martínez (Universidad de Zaragoza. Instituto Aragonés de Ciencias de la Salud).

Pilar Samper Villagrasa (Universidad de Zaragoza). 


\subsection{NOTIFICACIÓN A CENTROS}

Como ya se ha comentado en el punto anterior los componentes del grupo CORE, tras la aprobación del proyecto por el Instituto de Salud Carlos III, presentaron con detalle en cada uno de los centros de salud seleccionados los objetivos y el diseño del proyecto CALINA. En la misma notificación se invitaba formalmente a participar en bloque a todo el personal médico y de enfermería relacionado con la asistencia pediátrica en los centros de salud. La aceptación de participar era voluntaria con la posibilidad de retirarse cuando se quisiera.

Todos los que aceptaron participar se incorporaron como investigadores al grupo CALINA definitivo. Pertenecer al grupo de investigación CALINA implica la colaboración desde Atención Primaria en la recogida de datos $\mathrm{y}$, si el investigador lo desea, la posibilidad de participar en el análisis y divulgación de los resultados en diferentes foros.

\subsection{RONDA CALINA}

Varias semanas después de la notificación a los centros de Atención Primaria (durante enero de 2009), se realizó la «ronda CALINA» con los siguientes objetivos:

- Aclarar las dudas que sobre el proyecto se plantearan por parte del personal de los centros de Atención Primaria. 
- Evaluar la disponibilidad de material en cada centro para desarrollar el proyecto (báscula, medidor de longitud, lipómetro de compás...).

- Reclutar definitivamente al personal de enfermería y facultativos de pediatría que aceptaron participar.

- Organizar el reparto de tareas en las distintas fases del proyecto y el método de recogida de datos específico en cada centro.

\subsection{PARTICIPANTES Y CONSTITUCIÓN DEFINITIVA DEL GRUPO CALINA}

Tras la aceptación de participación en el estudio por parte del personal de Atención Primaria de los centros seleccionados, se constituyó definitivamente el grupo de investigación CALINA. El total de miembros participantes agrupados según su centro de trabajo se enumera a continuación.

\section{PERSONAL INVESTIGADOR PARTICIPANTE POR CENTROS}

\section{Centro de Salud Actur Norte-Oeste (Amparo Poch), Zaragoza}

- Bartolomé Lalanza, M. ${ }^{a}$ Lucía (Pediatra)

- Chicote Abadía, M. ${ }^{a}$ Begoña (Enfermera)

- Elviro Mayoral, Luis Carlos (Pediatra)

- Falcón Polo, Ángeles (Enfermera)

- Júdez Molina, Carmen (Enfermera) 
- Lostal Gracia, M. ${ }^{a}$ Isabel (Pediatra)

- Puig García, Carmen (Pediatra)

- Redondo Cuerpo, M. ${ }^{a}$ Victoria (Enfermera)

- Sánchez Zapater, Asunción (Pediatra)

- Viñas Viamonte, M. ${ }^{\mathrm{a}}$ Carmen (Enfermera)

\section{Centro de Salud Borja (Zaragoza)}

- Álvarez Otazu, M. ${ }^{a}$ Jesús (Enfermera)

- Lalana Josa, M. ${ }^{a}$ Pilar (Pediatra)

\section{Centro de Salud Delicias Sur, Zaragoza}

- Cabañas Bravo, M. J Jesús (Pediatra)

- Crespo Mainar, María Cleofé (Enfermera)

- Fuertes Domínguez, Amparo (Pediatra)

- García Sánchez, Nuria (Pediatra)

- Solans Bascuas, M. ${ }^{\text {a }}$ Teresa (Pediatra)

- Verón Jiménez, M. ${ }^{a}$ Asunción (Enfermera)

\section{Centro de Salud Jaca (Huesca)}

- Laín Ara, M. ${ }^{a}$ Lourdes (Enfermera)

- Lalaguna Puértolas, M. ${ }^{a}$ Jesús (Enfermera)

- Pinilla Fuentes, José Antonio (Pediatra)

- Puyuelo del Val, Pascual (Pediatra) 


\section{Centro de Salud Las Fuentes Norte, Zaragoza}

- Forés Catalá, M. ${ }^{a}$ Desamparados (Enfermera)

- Garín Moreno, Ana-Luz (Pediatra)

- Lambán Casamayor, Elisa (Enfermera)

- Marín Ibáñez, M. ${ }^{a}$ Pilar (Enfermera)

\section{Centro de Salud Perpetuo Socorro, Huesca}

- Alijarde Lorente, Roberto (Pediatra)

- Broto Cosculluela, Purificación (Enfermera)

- Fuertes Fernández-Espinar, Jorge (Pediatra)

- Pardos Martínez, Luis Carlos (Pediatra)

- Sebastián Bonel, M. ${ }^{a}$ Flor (Enfermera)

Centro de Salud Sagasta-Miraflores-Ruiseñores, Zaragoza

- Arana Navarro, Teresa (Pediatra)

- Cenarro Guerrero, Teresa (Pediatra)

- Fondevilla Pérez, M. ${ }^{a}$ Teresa (Enfermera)

- García Vera, César (Pediatra)

- Gila Gajón, M. ${ }^{a}$ Asunción (Enfermera)

- Sánchez Gimeno, Javier (Pediatra)

- Traín del Val, Ana (Enfermera) 


\section{Centro de Salud Tarazona (Zaragoza)}

- Baños Ledesma, M. ${ }^{a}$ Victoria (Enfermera)

- Martínez Espligares, Laura (Pediatra)

- Soria Martínez, Leticia (Enfermera)

\section{Centro de Salud Teruel Centro, Teruel}

- Adivinación Herrero, Dori (Enfermera)

- Galán Rico, José (Pediatra)

- Navarro Serrano, Eva María (Pediatra)

\section{Centro de Salud Teruel Ensanche, Teruel}

- Feliz de Vargas Pastor, Jesús (Pediatra)

- Muñoz Campos, Ana Isabel (Pediatra)

- Montaner Cosa, Mercedes (Enfermera)

\section{Centro de Salud Torrero-La Paz, Zaragoza}

- Balagué Clemos, Concepción (Enfermera)

- Cáncer Raginal, Rosa (Enfermera)

- Cebrián Gimeno, M. Inmaculada (Pediatra)

- Galbe Sánchez-Ventura, José (Pediatra)

- Zarazaga Germes, Gregorio (Pediatra) 


\section{Centro de Salud Valdefierro, Zaragoza}

- Atance Melendo, Esther (Enfermera)

- Blasco Pérez-Aramendia, M. ${ }^{a}$ Jesús (Pediatra)

- Gallego Pérez, Matilde (Enfermera)

- Marco Olloqui, Mónica (Pediatra)

\section{Centro de Salud Zuera (Zaragoza)}

- Martínez Bueno, José Luis (Enfermero)

- Traver Cabrera, Pilar (Pediatra)

\section{Instituto Aragonés de Ciencias de la Salud}

- Magallón Botalla, Rosa (Médico de Familia; Coordinadora del Grupo Aragonés de Investigación en Atención Primaria, GAIAP)

- Mengual Gil, José M. ${ }^{a}$ (Pediatra; Director del Área de Transferencia del Conocimiento)

\section{Universidad de Zaragoza}

- Álvarez Sauras, M. ${ }^{a}$ Luisa (Nutricionista)

- Ayerza Casas, Ariadna (Pediatra)

- Moreno Aznar, Luis A. (Prof. Titular Universidad)

- Muñoz Jalle, Elena (Pediatra)

- Olivares López, José Luis (Catedrático de Pediatría; Investigador Principal)

- Rodríguez Martínez, Gerardo (Prof. Titular Universidad; Pediatra)

- Samper Villagrasa, Pilar (Prof. Colaboradora; Pediatra) 


\section{TALLER DE CONSENSO Y ESTANDARIZACIÓN METODOLÓGICA}

Durante los días 16, 17 y 18 de febrero de 2009 se realizó un taller de consenso y estandarización metodológica de todos los documentos y técnicas contemplados en el proyecto CALINA. Se repasaron así mismo todas las variables que inicialmente se pretendían registrar en el estudio, descartando algunas que se consideraron inapropiadas para los objetivos y se añadieron otras que sí lo eran. Para las variables cuantitativas se consensuaron las unidades de medida y la metodología necesaria para su determinación. Para las variables cualitativas se definieron las categorías, su significado y la forma de registrarlas.

En el taller participaron todos los investigadores colaboradores (personal de pediatría y enfermería de los centros de salud incluidos en el proyecto CALINA) y contribuyeron activamente en la elaboración consensuada definitiva de los documentos que a continuación se detallan.

\subsection{INFORMACIÓN PARA EL PACIENTE}

Durante la primera visita a cada Centro de Salud, se dará a los padres un documento con información sobre el estudio CALINA (Anexo I). En este documento se explicará quién va a realizar el estudio, el objetivo que se pretende con el mismo, los datos que se van a recoger, el número de niños participantes, los beneficios que se obtendrán y la ausencia de riesgo para el 
bebé durante su desarrollo. También se hará constar en el documento informativo que la participación es voluntaria y se asegurará la confidencialidad de los datos. Todo ello se recogerá en un escrito que ocupará un folio e irá firmado y fechado por el sanitario que realice esta primera visita. En principio, era un documento más extenso y de comprensión dificultosa, por lo que se optó durante el taller de consenso por abreviarlo, sin perder información, facilitando así una lectura más sencilla.

\subsection{MODELO DE CONSENTIMIENTO INFORMADO PARA EL PACIENTE}

El consentimiento informado es un documento que se firmará por los padres o tutores en la primera visita del recién nacido al Centro de Salud (Anexo II). Su firma es obligatoria para la inclusión del niño en el estudio CALINA y para la recogida de cualquier variable contemplada en el proyecto. Este documento deberá estar perfectamente cumplimentado y firmado por uno de los dos padres y por el sanitario que realice esta primera visita. El documento deja claro que la participación es voluntaria y revocable; es decir, se puede retirar del estudio cuando se desee sin tener que dar explicaciones y sin que esto repercuta en los cuidados médicos posteriores.

\subsection{DATOS DEMOGRÁFICOS, OBSTÉTRICOS Y PERINATALES}

Los datos que se pretenden registrar en este apartado se obtendrán en la primera visita pediátrica en el centro de salud tras la salida del hospital (Anexo III). Se registrará, en primer lugar, la fecha de la primera consulta en 
el Centro de Salud, la fecha y hora de nacimiento, y con los datos anteriores se calculará la edad exacta de cada control clínico en el centro de salud. La variable sexo se señalará redondeando directamente en el anexo el que corresponda. Lugar de nacimiento y el hospital de procedencia, especificando si es público o privado. País de origen del padre y de la madre, anotando la etnia correspondiente, y la localidad donde habitan, especificando el Centro de Salud donde se realiza el estudio. Respecto a la etnia es importante, por la semejanza entre los apellidos de origen español, detallar los pertenecientes a la etnia gitana.

El nivel socioeconómico se investigará con: a) los estudios de los padres: superiores (universidad y ciclos formativos o FP de grado superior), medios (bachiller, y ciclos formativos o FP de grado medio), básicos (graduado escolar, ESO o equivalentes) y sin estudios; y b) la profesión de los mismos, de la madre nos interesa si su trabajo es fuera del domicilio, tipo de contrato: autónomo; cuenta ajena: fijo o eventual; jornada: entera, media o reducida, debiendo indicar el número de horas trabajadas, modalidad de jornada: intensiva, partida o a turnos. Se hará constar cuándo tiene previsto la madre acudir de nuevo al trabajo tras la maternidad, expresado en meses con un decimal.

Medidas de los padres, con los pesos expresados en kg, y las tallas en metros. También se registrará el peso materno previo a la gestación en kg y ganancia de peso durante la gestación en $\mathrm{kg}$. Se tomará nota de los datos referenciados por los padres y en caso de discrepancia o desconocimiento, si la familia lo permite, se les podrá pesar y tallar en la propia consulta. 
Hábitos tóxicos durante el embarazo en referencia al consumo de tabaco (sí o no) y número de cigarrillos al día, en el caso de que la respuesta sea afirmativa.

Control médico realizado durante el embarazo e incidencias ocurridas durante el mismo (preeclampsia, diabetes gestacional...) y si corresponde con un embarazo múltiple, anotando el número de fetos. Edad gestacional, en semanas cumplidas, a la que se produce el parto, modalidad de parto: eutócico, instrumental, cesárea, Test de Apgar al minuto y cinco minutos de vida e incidencias que ocurran en la etapa perinatal (desprendimiento de placenta...) y conlleven el ingreso hospitalario (en este caso habrá que dejar constancia de por qué ingresó). Número total de hijos de esa familia, incluyendo el propio hijo historiado.

Los datos antropométricos del niño al nacimiento incluirán el peso en gramos, longitud en centímetros con un decimal, perímetro craneal en centímetros con un decimal y peso al alta en gramos. Se deberá detallar la fecha de alta hospitalaria y hora de la misma. En cuanto a la alimentación, se indicará si existen o no antecedentes patológicos por parte de la madre o del recién nacido que contraindiquen o dificulten la lactancia materna, especificando cuáles son. Tipo de alimentación que está recibiendo el niño al salir del hospital (materna, fórmula o mixta); para registrar este dato, a la madre se le preguntará si el día último antes de salir del hospital tomó algún biberón de leche, y en el caso de que así sea (aunque sólo sea uno), se marcará la opción de lactancia mixta en el apartado de «alimentación al alta hospitalaria». Si en las siguientes revisiones únicamente toma pecho se 
considerará que la toma del biberón fue algo circunstancial, pero debe quedar reflejado.

\subsection{ENCUESTA MATERNA SOBRE LACTANCIA TRAS EL NACIMIENTO}

Se registrará la duración de la lactancia materna en cada uno de los hijos anteriores, en meses. Respecto a este hijo, se pretende conocer el momento en el que toma la decisión de ofrecerle lactancia materna: antes o durante el embarazo o bien después del parto; si ha recibido información acerca de la lactancia materna y cómo la ha recibido: a través de personal sanitario, de libros, prensa diaria o incluso por Internet o bien en los cursillos prenatales.

Cuando se pregunte por el motivo por el que alimenta a su hijo con lactancia materna, la contestación será libre ya que es una pregunta no dirigida en un principio; y si no sabe precisarlo, existen varias opciones que se le pueden indicar de manera dirigida: a) comodidad, b) tendencia actual, c) sentirse realizada, d) mayor contacto con mi hijo, e) me lo han aconsejado, f) ahorro económico, g) es el mejor alimento para mi hijo y h) otras razones. En el caso de que la pregunta sea dirigida se dejará constancia en el cuestionario.

El tiempo previsto de lactancia materna tiene varias opciones: menor de 4 meses, entre 4 y 6 meses, de 6 meses a 1 año o mayor de 1 año. También se especificará las razones para la decisión de ese tiempo previsto: a) hasta 
que empiece a trabajar, b) hasta que el niño quiera, c) hasta que pueda y d) otras.

Se preguntará por el motivo por el que alimenta a su hijo con leche de fórmula, lo contestarán también libremente. En el caso de no poder o saber precisarlo, se contempla la pregunta dirigida con las siguientes opciones: a) comodidad, b) me lo han aconsejado, c) es el mejor alimento para mi hijo, d) seguir tendencia actual y e) otras razones. En el caso de que la pregunta sea dirigida se dejará constancia en el cuestionario.

Se señalará si ha influido la familia o el personal sanitario en la decisión del tipo de lactancia que ofrece a su hijo.

\subsection{SEGUIMIENTO DE LA ALIMENTACIÓN}

\section{Periodo de lactancia}

Las preguntas correspondientes a este apartado se realizarán en todos los casos tras el destete definitivo.

Como tiempo de permanencia con lactancia materna exclusiva (expresado en meses o días) se contempla el período de alimentación con leche humana exclusivamente o de manera predominante (toma muy ocasional de infusiones o líquidos no lácteos). La duración de la lactancia materna será interpretada como el momento definitivo del destete (en meses con decimales). El motivo de abandono de la lactancia materna será preguntado de forma no dirigida, pudiendo marcar varias posibilidades: a) trabajo, b) enfermedad de la madre o el hijo, c) el niño no quiere, d) la madre 
no quiere, e) escasa ganancia ponderal, f) se queda con hambre, g) pérdida de peso, h) hipogalactia o escasa secreción láctea, i) problemas con la lactancia (grietas, mastitis, técnica, ...), y j) otras (debiendo especificar). Si la pregunta se hace dirigida deberá señalarse este hecho. Se detallará quién decidió el momento del destete: la madre, el padre, los abuelos, el personal sanitario, el propio bebé. Se comprobará si la madre consultó con algún grupo de apoyo a la lactancia materna y en qué momento. Así mismo, en el caso de que la madre haya utilizado métodos de extracción de leche materna, se especificará el motivo y los meses de vida en que sucedió. También se indicará si se usaron métodos de conservación de la leche materna, detallando si fue en el frigorífico o congelador y cuándo sucedió este hecho.

Respecto a la leche de fórmula, se anotará el tiempo a partir del cual empezó a ofrecerle cualquier tipo de leche de fórmula, así como el motivo de este cambio (respuesta no dirigida por el encuestador) y la persona que decidió su comienzo.

\section{Alimentación complementaria}

Respecto a la alimentación complementaria, se preguntará en las visitas sucesivas y a criterio temporal del profesional sanitario la edad de introducción de los siguientes alimentos no lácteos: a) cereales sin gluten, b) fruta, c) verdura, d) carne, e) cereales con gluten, f) pescado, g) huevo, h) yogur, i) legumbre. También se deberá anotar la edad en la que el niño comienza a comer a trocitos (meses con un decimal). 
Los hábitos alimenticios del niño se preguntarán a los 24 meses. Los apartados contemplados son los siguientes: a) si come a trocitos, b) si come solo, c) el número de tomas que hace al día, incluyendo las tomas de leche líquida, d) si come de todo lo que le corresponde a su edad. Finalmente se anotará si la madre considera que su niño/a come poco o es mal comedor.

\subsection{DESARROLLO PSICOMOTOR}

Dentro del apartado del desarrollo psicomotor se anotará la edad a la que adquiere los siguientes ítems, a lo largo del primer año de vida: a) inicio de la sonrisa social, expresada en semanas, b) sostén cefálico, en meses con un decimal, c) sedestación completa sin ayuda y estable, en meses con un decimal, d) pinza superior, en meses con un decimal, e) deambulación solo, siendo capaz de dar 5 pasos seguidos sin ayuda, en meses con un decimal.

Se anotará si acude a guardería y la edad de su inicio, expresada en meses con un decimal.

\subsection{ANTROPOMETRÍA Y LACTANCIA EN LAS REVISIONES}

En cada una de las revisiones rutinarias en la consulta de pediatría de Atención Primaria se realizarán las medidas antropométricas y el registro de la lactancia. Las revisiones son las correspondientes al programa del niño sano a los 15 días de edad, 1, 2, 3, 4, 6, 9, 12, 15, 18 y 24 meses. Las medidas 
antropométricas que se contemplan son: peso, longitud, perímetro cefálico, perímetro abdominal, perímetro braquial, pliegues cutáneos (bicipital, tricipital, suprailiaco, subescapular). En cada una de estas visitas se anotará la fecha y la edad del niño expresada en meses y semanas, o solo en días en el caso de las primeras. También en cada una de las sucesivas visitas se preguntará por el tipo de lactancia recibida por el niño el día anterior (y solo el día anterior); de esta manera, se hace más fácil la pregunta y se obtiene una visión general más exacta de la realidad. Las opciones son: lactancia materna y/o lactancia artificial (anotando el tipo de leche de fórmula). También se dejará constancia de si el día anterior ha tomado algún otro líquido (infusiones, zumos) o sólido, anotando este hecho en el hueco que queda en el anexo, debajo de cada revisión y escrito a mano hasta los 6 meses (Anexo III).

En los centros que excepcionalmente no realizan alguna de las revisiones planificadas (por ejemplo la del tercer mes) no tendrán que incorporarla en su programa.

Las medidas antropométricas se realizarán por el personal habitual de cada centro participante que ya está debidamente entrenado, con el niño desnudo, en condiciones ambientales adecuadas y siempre en el mismo horario (mañanas o tardes). Se comprobará antes del comienzo del estudio que el material disponible es adecuado y fiable:

- El peso se obtendrá con el pesabebés disponible en cada consulta (precisión $10 \mathrm{~g}$ ), previamente calibrado, con el niño desnudo y en la postura adecuada según la edad. 
- La longitud se realizará con el tallímetro disponible en cada consulta, con una precisión de $0,1 \mathrm{~cm}$. El niño se colocará siempre en decúbito prono sobre la lámina horizontal, con el vértex en contacto con el límite fijo vertical, sujetando la cabeza para evitar desplazamientos. Con ayuda de otra persona, el explorador extenderá al menos una de las extremidades inferiores (mejor las dos), evitando que flexione las rodillas, hasta llegar a contactar el talón con la otra parte rígida, vertical y móvil del medidor. Finalmente se comprobará antes de obtener la medida que la cabeza no se ha separado de la lámina vertical.

- El perímetro cefálico se medirá colocando una cinta inextensible por la parte inferior del frontal, sobre los arcos ciliares y por la parte más saliente del occipital, de manera que se determine la circunferencia máxima craneal, con una precisión de $0,1 \mathrm{~cm}$.

- El perímetro abdominal se determinará mediante una cinta inextensible colocada justo por encima del ombligo, con una precisión de $0,1 \mathrm{~cm}$.

- El perímetro braquial se obtendrá en la línea perpendicular al eje del brazo izquierdo, en el punto equidistante de ambos extremos del húmero (acromion y olecranon), midiendo en ese punto la circunferencia máxima, con una precisión de $0,1 \mathrm{~cm}$.

- Los pliegues cutáneos se medirán con un calibrador de presión constante entre ramas $\left(10 \mathrm{gm} / \mathrm{cm}^{2}\right)$ y graduación mínima de $0,2 \mathrm{~mm}$, con intervalo de lectura entre 2 y $20 \mathrm{~mm}$. El error de precisión es del 5 $\%$ en personal experimentado, pero puede llegar al $10 \%$. El calibrador 
utilizado es el metálico de la marca Holtain. Para realizar las mediciones se debe coger el calibrador con la mano dominante y, con los dedos índice y pulgar de la mano no dominante, se pellizca muy próximo al lugar donde se desea realizar la medición. El pellizco sólo debe contener tejido subcutáneo y no tejido muscular. El calibrador se colocará sobre el área pellizcada y se esperará a que la aguja marcadora se estabilice ( 2 segundos aprox.). Se deben realizar tres mediciones sucesivamente y tomar la media de las tres determinaciones. El pliegue subescapular se valorará en el punto localizado por debajo y por dentro de la escápula izquierda; el pliegue suprailiaco en el punto localizado en la zona inmediatamente superior a la cresta iliaca, en la línea medio axilar anterior; el pliegue tricipital en el punto medio entre el acromion y el olecranon, en la parte posterior del brazo; y finalmente, el pliegue bicipital en el mismo nivel que el tricipital, pero en la cara anterior del brazo. 


\title{
7.8. DOCUMENTOS CONSENSUADOS FINALES
}

\author{
Anexo I \\ INFORMACIÓN PARA EL PACIENTE
}

Apreciado Sr./Sra.:

El Instituto de Ciencias de la Salud del Gobierno de Aragón va a realizar el estudio financiado por el Instituto de Salud Carlos III titulado:

\section{CRECIMIENTO Y ALIMENTACIÓN DURANTE LA LACTANCIA Y LA PRIMERA INFANCIA EN NIÑOS ARAGONESES (CALINA)}

Antes de confirmar su participación es importante que conozca el estudio. Por favor, lea este documento y haga todas las preguntas que puedan surgirle.

Objetivo del estudio: Valorar el crecimiento y las pautas de alimentación de los niños aragoneses hasta los 24 meses de edad.

Número de bebés para el estudio: 2300 nacidos durante el año 2009, que acudirán a las revisiones programadas del niño sano en Atención Primaria.

Datos a recoger en el estudio en los niños participantes:

- Datos demográficos de los padres y los bebés.

- Historia clínica obstétrica y perinatal.

- Datos sobre alimentación.

- Antropometría: Peso, longitud, perímetros corporales, pliegues cutáneos.

Los datos se recogerán en las visitas programadas de revisión del niño sano.

Beneficios: Además de los beneficios que aportarán los resultados del estudio, durante este periodo su bebé estará muy controlado respecto a su crecimiento.

\section{Riesgos: NO EXISTE NINGÚN RIESGO PARA SU BEBÉ.}

Participación voluntaria: Usted decide si quiere participar o no. Incluso si decide participar, puede retirarse del estudio en cualquier momento sin tener que dar explicaciones. En ningún caso esto afectará su atención médica posterior.

Confidencialidad: Los datos serán protegidos de usos no permitidos y se respetará la confidencialidad de los mismos (Leyes Orgánicas 15/1999 y 41/2002). La información generada será estrictamente confidencial, permitiéndose, sin embargo, su inspección por las Autoridades Sanitarias. El estudio se llevará a cabo siguiendo las normas deontológicas reconocidas y cumpliendo la legislación vigente (Real Decreto 223/2004 y Ley 14/2007 de Investigación Biomédica).

Preguntas/Información: En las sucesivas visitas podrá preguntar sus dudas y se le informará progresivamente de los datos obtenidos.

Nombre y firma del Sanitario

Fecha. ... 
Anexo II

MODELO DE CONSENTIMIENTO INFORMADO

PARA EL PACIENTE

\section{Título del PROYECTO:}

\section{CRECIMIENTO Y ALIMENTACIÓN DURANTE LA LACTANCIA Y LA PRIMERA INFANCIA EN NIÑOS ARAGONESES (CALINA)}

Yo, (nombres y apellidos)

He leído la hoja de información que se me ha entregado.

He podido hacer preguntas sobre el estudio.

He recibido suficiente información sobre el estudio.

He hablado con: .(nombre del Sanitario)

Comprendo que mi participación es voluntaria.

Comprendo que puedo retirarme del estudio:

1) cuando quiera

2) sin tener que dar explicaciones

3) sin que esto repercuta en mis cuidados médicos

Presto libremente mi conformidad para participar en el estudio.

Doy mi conformidad para participar en este proyecto de investigación clínica, y soy consciente de que este consentimiento es revocable.

Firma del participante:

Fecha:

He explicado la naturaleza y el propósito del estudio al paciente mencionado.

Firma del Sanitario:

Fecha: 


\section{Anexo III}

\section{NOMBRE:}

\section{DATOS DEMOGRÁFICOS, OBSTÉTRICOS Y PERINATALES:}

Fecha de nacimiento:

Lugar nacimiento:

País de origen del padre:

Localidad donde habita:
Hora de nacimiento

Sexo: Varón Mujer

Hospital público privado

País de origen de la madre:

Etnia:

Control gestacional: Sí NO

Incidencias gestacionales:

Edad gestacional (semanas): Tipo de parto: Eutócico Instrumental Cesárea

Incidencias perinatales:

Test de Apgar: 1' 5, Ingreso: SÍ NO Embarazo múltiple: SÍ NO

Número de hijos vivos de la madre (incluido el actual):

Antecedentes maternos/niño que contraindiquen/dificulten la LM:

Peso RN (g):

Longitud RN (cm):

Perímetro craneal RN (cm):

Fecha alta hospitalaria:

Hora alta:

Peso al alta hospitalaria $(\mathrm{g})$ :

Alimentación al alta hospitalaria: materna mixta fórmula

Fecha primera consulta en centro de salud:

Peso materno previo gestación $(\mathrm{kg})$ : $\quad$ Ganancia peso madre gestación $(\mathrm{kg})$ :

Talla madre $(\mathrm{m})$ :

Peso padre $(\mathrm{kg})$ :

Talla padre (m):

Fumó durante el embarazo: SÍ NO

N. ${ }^{\circ}$ de cigarrillos/día:

Estudios madre: Ninguno Básicos Medios Superiores Profesión madre:

Estudios padre: Ninguno Básicos Medios Superiores Profesión padre:

Madre trabaja fuera del domicilio: SÍ NO

¿Cuándo volverá al trabajo tras maternidad? (mes con un decimal)

Tipo de contrato laboral madre a) autónomo

b) cuenta ajena: fijo / eventual

c) jornada: entera / media / reducida (horas trabajo)

d) jornada: intensiva / partida (mañana y tarde) / turnicidad 


\section{NOMBRE:}

\section{ENCUESTA MATERNA SOBRE LACTANCIA TRAS EL NACIMIENTO}

- Duración lactancia materna hijos anteriores (meses): Hijo 1 Hijo 2 Hijo 3

- Momento toma decisión LM: antes embarazo / durante embarazo / después parto

- Ha recibido información acerca de LM: SÍ NO
1. Personal sanitario
2. Libros, prensa, Internet
3. Cursillos prenatales

- Motivos de decisión de LM (pregunta no dirigida): 1. comodidad 2. tendencia actual 3. sentirse realizada 4. mayor contacto con mi hijo 5. me lo han aconsejado
6. ahorro económico
7. es el mejor alimento para mi hijo
8. otros

- Motivos de decisión de LA (pregunta no dirigida): 1. comodidad 2. me lo han aconsejado
3. mejor alimento para mi hijo
4. seguir tendencia
5. otros

- Tiempo previsto de lactancia materna: <4 meses entre 4-6 meses $\quad>6$ meses

$$
6 \text { meses-1 año >1 año }
$$

- Por qué de ese tiempo previsto:

1) hasta que empiece a trabajar
2) hasta que el niño quiera
3) hasta que pueda
4) otras

- Influencia de la familia en el tipo de lactancia: SÍ NO

- Influencia del personal sanitario en decisiones sobre lactancia: SÍ NO 


\section{NOMBRE:}

\section{SEGUIMIENTO DE LA ALIMENTACIÓN (hacerla tras el destete)}

- Lactancia materna exclusiva durante (meses o días)

- Infusiones o líquidos no lácteos habituales: SÍ NO

- Lactancia materna hasta qué edad (momento del destete en meses con decimales)

- Motivo abandono de lactancia materna (se pueden marcar varias; pregunta no dirigida)

$$
\begin{aligned}
& \text { 1= Trabajo 2= Enfermedad madre/hijo } \\
& 3=\mathrm{El} \text { niño no quiere } \quad 4=\mathrm{La} \text { madre no quiere } \\
& 5=\text { Escasa ganancia ponderal } \quad 6=\text { Se queda con hambre } \\
& \text { 7= Perdía peso } \quad 8=\text { Hipogalactia/escasa secreción } \\
& \text { 9= Problemas lactancia (grietas, mastitis, técnica...) } \\
& 10=\text { Otros (especificar) }
\end{aligned}
$$

- Quién decidió el momento del destete (madre, padre, abuelos, personal sanitario, bebé)

- ¿Consultó con algún grupo de apoyo a la LM? Sí/no ¿Cuándo?

— ¿Se utilizaron métodos de extracción de LM si dificultades o ausencias madre?

$$
\text { Sí / no Especificar cuándo (meses de vida): la causa: }
$$

- ¿Se utilizaron métodos de conservación de LM? Sí / no Frigo / congelador ¿Cuándo?

- ¿Cuándo empezó a tomar cualquier leche de fórmula?

- Motivo del comienzo con leche de fórmula (pregunta no dirigida)

- ¿Quién decidió el comienzo con leche de fórmula? 


\section{NOMBRE:}

\section{ALIMENTACIÓN COMPLEMENTARIA}
- Edad de introducción de alimentos: Cereales sin gluten:
Fruta: Verdura:
Carne:
Cereales con gluten:
Pescado: Huevo:
Yogur:
Legumbre:
Trocitos de comida:

\section{HÁBITOS ALIMENTICIOS (rellenar a los 24 meses)}

- El niño a los 24 meses, ¿toma trozos? Sí / no ¿Come solo? Sí / no

- ¿Cuántas veces come al día a los 24 meses? (incluyendo tomas de leche)

- ¿Come de todo? Sí / no

- ¿Considera la madre que come poco o que es mal comedor? Sí / no

- ¿Acude a la guardería? Sí / no ¿Desde qué edad?

\section{DESARROLLO PSICOMOTOR}

- Sonrisa social (semanas)

- Sostén cefálico (meses con decimales)

- Sedestación completa estable (meses con decimales)

- Pinza superior (meses con decimales)

- Deambulación (5 pasos) (meses con decimales) 


\section{NOMBRE:}

\section{ANTROPOMETRÍA Y LACTANCIA EN LAS REVISIONES}

15 DÍAS Fecha:

Peso (g) Longitud (cm)

Pliegues cutáneos (mm): bicipital

Lactancia materna: Sí / No
Especificar días de vida:

PC $(\mathrm{cm}) \quad$ Per. abdominal $(\mathrm{cm}) \quad$ Per. Brazo $(\mathrm{cm})$

$$
\text { tricipital subescapular suprailiaco }
$$

L. artificial: Sí / No Tipo de leche:

\section{MES Fecha:}

Peso (g) Longitud (cm)

Pliegues cutáneos (mm): bicipital

Lactancia materna: Sí/ No
Edad (meses + semanas)

PC $(\mathrm{cm}) \quad$ Per. abdominal $(\mathrm{cm}) \quad$ Per. Brazo $(\mathrm{cm})$

$$
\text { tricipital subescapular suprailiaco }
$$

L. artificial: Sí / No Tipo de leche:
2 MESES Fecha:

Peso (g) Longitud (cm)

Pliegues cutáneos (mm): bicipital

Lactancia materna: Sí / No
Edad (meses + semanas)

PC (cm) Per. abdominal (cm) Per. Brazo (cm)

$$
\text { tricipital subescapular suprailiaco }
$$

L. artificial: Sí / No Tipo de leche:

\section{MESES Fecha:}

Peso (g) Longitud (cm)

Pliegues cutáneos (mm): bicipital

Lactancia materna: Sí / No

L. artificial: Sí / No
Edad (meses + semanas)

PC $(\mathrm{cm}) \quad$ Per. abdominal $(\mathrm{cm}) \quad$ Per. Brazo $(\mathrm{cm})$ tricipital subescapular suprailiaco 
4 MESES Fecha:

Peso (g) Longitud $(\mathrm{cm})$

Pliegues cutáneos (mm): bicipital

Lactancia materna: Sí / No
Edad (meses + semanas)

PC $(\mathrm{cm}) \quad$ Per. abdominal $(\mathrm{cm}) \quad$ Per. Brazo $(\mathrm{cm})$

$$
\text { tricipital subescapular suprailiaco }
$$

L. artificial: Sí / No Tipo de leche:

\section{MESES Fecha:}

Peso (g) Longitud (cm)

Pliegues cutáneos (mm): bicipital

Lactancia materna: Sí / No
Edad (meses + semanas)

$$
\text { PC }(\mathrm{cm}) \quad \text { Per. abdominal }(\mathrm{cm}) \quad \text { Per. Brazo }(\mathrm{cm})
$$

$$
\text { tricipital subescapular suprailiaco }
$$

L. artificial: Sí / No Tipo de leche:
9 MESES Fecha:

Peso (g) Longitud (cm)

Pliegues cutáneos (mm): bicipital

Lactancia materna: Sí / No
Edad (meses + semanas)

PC (cm) Per. abdominal $(\mathrm{cm}) \quad$ Per. Brazo $(\mathrm{cm})$

$$
\text { tricipital subescapular suprailiaco }
$$

L. artificial: Sí / No Tipo de leche:
12 MESES Fecha:

$$
\text { Peso (g) Longitud (cm) }
$$

Pliegues cutáneos (mm): bicipital

Lactancia materna: Sí / No
Edad (meses + semanas)

$$
\begin{array}{ccc}
\text { PC }(\mathrm{cm}) & \text { Per. abdominal }(\mathrm{cm}) & \text { Per. Brazo }(\mathrm{cm}) \\
\text { tricipital } & \text { subescapular } & \text { suprailiaco }
\end{array}
$$

L. artificial: Sí / No Tipo de leche: 
15 MESES Fecha:

Peso (g) Longitud (cm)

Pliegues cutáneos (mm): bicipital

Lactancia materna: Sí / No

Edad (meses + semanas)

PC $(\mathrm{cm}) \quad$ Per. abdominal $(\mathrm{cm}) \quad$ Per. Brazo $(\mathrm{cm})$

$$
\text { tricipital subescapular suprailiaco }
$$

L. artificial: Sí / No Tipo de leche:

18 MESES Fecha:

Peso (g) Longitud (cm)

Pliegues cutáneos (mm): bicipital

Lactancia materna: Sí / No

Edad (meses + semanas)

24 MESES Fecha:

Peso (g) Longitud $(\mathrm{cm})$

Pliegues cutáneos (mm): bicipital

Lactancia materna: Sí / No
Edad (meses + semanas)

PC $(\mathrm{cm}) \quad$ Per. abdominal $(\mathrm{cm}) \quad$ Per. Brazo $(\mathrm{cm})$

tricipital subescapular suprailiaco

L. artificial: Sí / No Tipo de leche: 


\section{CRONOGRAMA FINAL REAL}

El proyecto CALINA se divide en tres etapas (ver esquema del cronograma):

La primera etapa del proyecto CALINA que comienza en enero de 2009 con el reclutamiento de los Centros de Salud que van a participar, y acaba en febrero de ese mismo año con el taller de consenso y estandarización metodológica. Durante estos dos meses se constituye el grupo CALINA definitivo y se perfilan los documentos y técnicas que se van a emplear en el estudio.

La segunda etapa comienza en marzo de 2009 y dura hasta febrero de 2012 (ambos inclusive). Durante este largo período de tiempo se desarrolla el trabajo de campo del estudio longitudinal. Dentro de esta etapa se distinguen varias partes:

-Reclutamiento y seguimiento durante 24 meses de cada uno de los niños, de esta manera, a los niños que nazcan en marzo de 2009 se les realizará el seguimiento del estudio hasta marzo de 2011.

-Evaluación de la precisión inter e intraobservador en varios momentos del estudio.

- Recogida en cada Centro de Salud de los cuestionarios y datos de la exploración antropométrica obtenidos del seguimiento de los niños.

-Registro y codificación en la base de datos de las variables recogidas. 
La tercera etapa empieza en septiembre de 2010 y consiste en el análisis y difusión de resultados. Esta etapa no tiene un límite determinado y continuará mientras existan datos que discutir y comunicar obtenidos del proyecto CALINA. 


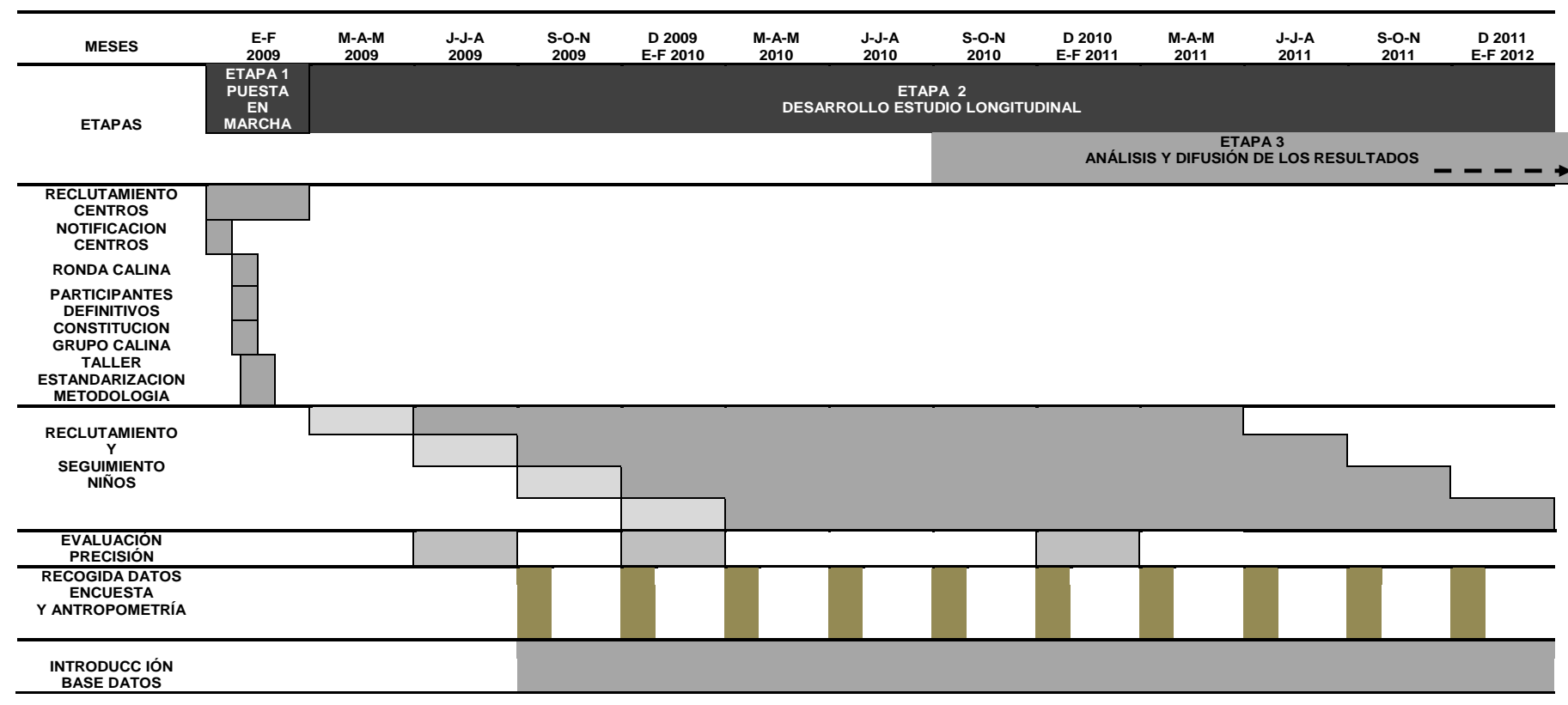





\section{ÍNDICE}

$\begin{array}{ll}\text { AUTORES COLABORADORES } & 9\end{array}$

$\begin{array}{ll}\text { PREFACIO } & 17\end{array}$

1 MEMORIA DEL PROYECTO CALINA 19

1.1. Introducción y justificación $\quad 19$

- Crecimiento

- Tablas y gráficas de crecimiento

- Alimentación del lactante. Lactancia materna

- Justificación

1.2. Objetivos 28

$\begin{array}{ll}\text { 1.3. Metodología } & 29\end{array}$

- Diseño

- Sujetos de estudio

- Tamaño muestral

1.4. Variables contempladas inicialmente 31

1.5. Recogida y análisis de datos $\quad 32$

1.6. Aspectos éticos 34

1.7. Limitaciones del estudio $\quad 34$

1.8. Cronograma y distribución de las tareas 36

1.9. Bibliografía 39

2 EXPERIENCIA DEL EQUIPO INVESTIGADOR SOBRE EL TEMA 43

- Líneas de investigación

3 RELEVANCIA DEL PROYECTO 47

- Impacto clínico, asistencial y/o desarrollo tecnológico

- Impacto bibliométrico de las publicaciones 
5.1. Informe de viabilidad del proyecto 51

5.2. Comité Ético de Investigación Clínica de Aragón (CEICA) 52

5.3. Financiación. Informe del Instituto de Salud Carlos III

6 RECLUTAMIENTO DE CENTROS PARTICIPANTES EN EL PROYECTO MUESTRA DEL PROYECTO CALINA 55

6.1. Grupo CORE

6.2. Notificación a centros $\quad 57$

6.3. Ronda CALINA $\quad 57$

6.4. Participantes y constitución definitiva del grupo CALINA 58

7 TALLER DE CONSENSO Y ESTANDARIZACIÓN METODOLÓGICA 63

7.1. Información para el paciente $\quad 63$

7.2. Modelo de consentimiento informado para el paciente 64

7.3. Datos demográficos, obstétricos y perinatales $\quad 64$

7.4. Encuesta materna sobre lactancia tras el nacimiento 67

7.5. Seguimiento de la alimentación $\quad 68$

$\begin{array}{ll}\text { 7.6. Desarrollo psicomotor } & 70\end{array}$

7.7. Antropometría y lactancia en las revisiones $\quad 70$

- Edad de las revisiones

- Lactancia

- Peso, longitud, pliegues cutáneos, circunferencias...

7.8. Documentos consensuados finales $\quad 74$

8 CRONOGRAMA FINAL REAL 83 
ISBN 978-84-92774-36-4

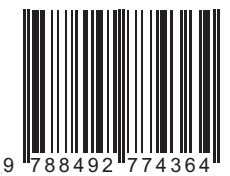

Colabora

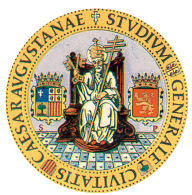

Financia

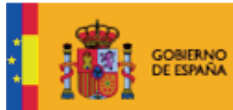

MINISTEROO

DE CIENCU
Organiza

GOBIERNO DE ARAGON Departamento de Salud
y Consumo 